\title{
La caliza de Morata de Tajuña, Comunidad de Madrid: una piedra tradicional de construcción en la capital a principios del siglo $\mathrm{XX}$
}

\author{
E. M. Pérez-Monserrat ${ }^{(1)}, C$. Peropadre ${ }^{(2,3)}$, R. Fort ${ }^{(1)}$ y $M^{a}{ }^{3}$. Varas-Muriel ${ }^{(1,4)}$ \\ (1) Instituto de Geociencias IGEO (CSIC, UCM). Doctor Severo Ochoa 7, Ciudad Universitaria, 28040 Madrid \\ empmon@geo.ucm.es, rafael.fort@csic.es \\ (2) Departamento de Estratigrafía, Facultad Ciencias Geológicas (UCM). José Antonio Nováis 12, 28040 Madrid \\ (3) Inypsa informes y proyectos S.A. General Díaz Porlier 49, 28001 Madrid \\ cperopadre@gmail.com \\ (4) Departamento de Petrología y Geoquímica, Facultad Ciencias Geológicas (UCM). José Antonio Nováis 12, 28040 Madrid \\ mjvaras@geo.ucm.es
}

\begin{abstract}
RESUMEN
A partir del estudio sobre la procedencia y la calidad de la caliza empleada en la construcción -a principios del siglo XX- de un emblemático inmueble madrileño, se analizan los principales factores que en esta época favorecieron su utilización. Se trata de una piedra tradicionalmente empleada en la región pero apenas conocida en la capital y que por entonces se explotaba en las canteras Cornicabra, ubicadas en el paraje del Valhondo de Morata de Tajuña (Comunidad de Madrid). Por un lado, esta caliza resultaba una piedra económicamente muy ventajosa, debido principalmente a la favorable situación por entonces de las canteras y de las vías de comunicación. Por otro, debido a su origen geológico y posteriores procesos diagenéticos, presenta una muy elevada calidad y durabilidad, resultando un material muy apropiado para configurar elementos portantes especialmente resistentes a la acción del agua y al propio paso del tiempo. Considerando una época en la que la piedra tradicional de la región estaba siendo sustituida por piedras procedentes de otros lugares, el prestigio del que históricamente ha disfrutado la caliza extraída en las canteras de Colmenar de Oreja y el efímero empleo en la capital de la caliza de Morata de Tajuña como piedra de cantería, el carácter tradicional de la caliza objeto de estudio resulta ciertamente excepcional.
\end{abstract}

Palabras clave: canteras de Cornicabra, diagénesis, litofacies, Mioceno, paraje del Valhondo.

\section{Limestone from Morata de Tajuña, Community of Madrid (Spain): used as a traditional building stone in the city of Madrid in the early $20^{\text {th }}$ century}

\author{
ABSTRACT
}

Based on the study of the limestone used in an emblematic building in Madrid, this analysis explores the factors that favoured the use of this limestone which was scarcely known in the city until the early twentieth century. This local stone was extracted from the Cornicabra quarry at Valhondo, near Morata de Tajuña in the Spanish province of the Comunity of Madrid. The Morata de Tajuna limestone was a more economical option at the beginning of the century, due primarily to the favourable status of the quarries and existing communications with the capital city. In addition, its high quality and durability afforded the stone by its genesis and diagenesis made it highly suitable for use in water- and wear-resistant structural members. The prestige historically accorded the limestone quarried at Colmenar de Oreja, the tendency at the time to replace it with stones from other areas and the ephemeral use of Morata de Tajuña limestone in the city of Madrid, render its short-lived prevalence as a traditional building stone truly exceptional.

Keywords: Cornicabra quarries, diagenesis, litofacies, Miocene, Valhondo natural area. 
Pérez-Monserrat, E. M., et al., 2017. La caliza de Morata de Tajuña, Comunidad de Madrid:... Boletín Geológico y Minero, 128 (4): $963-988$

\section{ABRIDGED ENGLISH VERSION}

\section{Introduction and Methodology}

Research into the origin and quality of the traditional stone used in the building heritage reveals a variety of factors which, taken as a whole, can be seen to have conditioned selection and use in a given age and for a specific constructional purposes.

One of the most prominent domains constituting the region of Madrid's geological substrate is the Basin of Madrid (Fig. 2a). Its Late Miocene Unit comprises a mosaic of fluvial-lacustrine systems where a number of the lithofacies present in the series developed (Fig. 2b). Late Miocene fluvial-lacustrine carbonates have traditionally been quarried as a building stone in the region, with the major quarries located in the high moors around Chinchón-Colmenar de Oreja and Arganda-Morata de Tajuña (Fig. 3). Given the quality, purity and whiteness of the Colmenar de Oreja material, the most highly reputed quarries were located there and the term Colmenar stone was used to designate the Miocene limestone quarried in and around that town.

This paper draws from the study conducted on the precedence and quality of the limestone used to build the former Hospital of Maudes (1909-1916), built by Antonio Palacios Ramilo (1874-1945) near the current Cuatro Caminos roundabout in Madrid (Fig. 1). This stone was sourced from the Cornicabra quarries in the Valhondo natural area in Morata de Tajuña. The present historic study of the primary reasons for its use as a building stone in the early twentieth century in the city of Madrid, in lieu of the prestigious Colmenar limestone, led to the identification of a quarry front in the former Cornicabra quarries which is nowadays abandoned. The front is described and the petrographical features (texture and composition) of the limestone from the outcrop and the building were compared. The latter was also characterised petrophysically with nondestructive (NDT) and standardised tests (Table 1). The petrographic and petrophysical characterisation of the limestone used to build the hospital denoted mechanical and hydric performance comparable to or even better than that found for the Colmenar de Oreja material.

\section{Results and discussion}

Morata de Tajuña versus Colmenar de Oreja limestone in the early twentieth century

The Colmenar de Oreja quarries were abandoned in the late nineteenth and early twentieth centuries more for economic considerations than for depletion of the stone. The railway network in place in the country at the time made the use of limestone from further away much more cost-efficient. Although the quarries were reactivated after the construction of a railway to Colmenar de Oreja in 1903, stone from outside the province continued to be used due to its lower cost. Whilst limestone continued to be quarried at Colmenar de Oreja in the early twentieth century, output was less than in the past.

By that time, the Morata de Tajuña limestone was well known as a prime material for the lime and cement manufactured at nearby Valhondo. A document located in the Fundación de los Ferrocarriles Españoles Historic Archives custodied by the Foundation's Madrid Railway Museum, provided insight into the intense quarrying conducted at Cornicabra quarries at the time and the potential of its limestone, primarily as a masonry stone. Cornicabra limestone was highly competitive in the early twentieth century. On the one hand, the near absence of any need for land clearing made quarrying simpler than at the Colmenar de Oreja quarries. On the other, the railway line, with its stop alongside the Conicabra quarries, lowered shipping costs (Figs. 4, 5 and 6, Table 2), making this limestone less expensive than others quarried at the time, both within and outside the province. The use of Cornicabra limestone as a building stone in the city of Madrid peaked approximately between 1905 and 1925.

Comparison of the outcrop with the building limestone and the quality of the Morata de Tajuña stone

The series established at the former quarry front, with an upward increase in stratum thickness, comprises primarily oncolitic limestone with high sparry calcite and tuff content (Fig. 7). A lower bed was found to consist of oncolitic greyish-beige limestone with well cemented bioclasts, followed by a bed of pale marlstone, alternating with tuffaceous limestone, beige oncolitic limestone and greyish, oncolitic limestone with sparry calcite; and an upper a bed containing pale oncolitic limestone and a dark, sparry calcite-bearing oncolitic limestone. The beige or yellowish and highly cemented limestone in the building is either micritic or formed by biogenic constructions -primarily stromatolitic-, respectively associated with the uniform and the stromatolitic-oncolitic facies (Fig. 8). The petrographic characterisation of the samples taken at the quarry and from the building revealed their similarity (Fig. 9). This comparative study further confirmed that the limestone in the former hospital was sourced from the Cornicabra quarries, as recorded in the documents consulted. 
Pérez-Monserrat, E. M., et al., 2017. La caliza de Morata de Tajuña, Comunidad de Madrid:... Boletín Geológico y Minero, 128 (4): $963-988$

The Morata de Tajuña (quarry and hospital) limestone owes its petrological properties to the environment prevailing during its formation and the diagenetic processes taking place in the basin, which gave rise to a very hard and resistant stone, well suited for use in bearing members. In addition to its high compressive strength, the stone exhibits good hydric performance, which further enhances its durability. Its most prominent textural characteristics include an abundance of bioclastic moulds, denoting porosity liable to cementation, and the absence of prevalent orientations. Cementation, the most prominent diagenetic process, reduces primary porosity, especially the intercrystalline porosity associated with micrite.

The high values of ultrasonic wave propagation $(V p>5500 \mathrm{~m} / \mathrm{s})$ and surface hardness $(R \approx 56)$ observed in this study attested to both the good mechanical performance of this limestone and its close compactness. The similar anisotropic index values symptomatic of the absence of a prevalent orientation would have constituted a worksite advantage, for the stones did not have to be positioned in any specific direction. The narrow difference between the real and bulk density values was indicative of low effective porosity. The water resistance of the limestone was confirmed by the clearly unimodal porosimetry curves, with the concentration of most of the pores in the 0.01-0.1 m range (Fig. 10). The resulting low permeability coefficient value $(0.06$ $\mathrm{g} / \mathrm{m}^{2} \cdot 24 \mathrm{~h} \mathrm{mmHg}$ ) revealed that the stone is highly resistant to water vapour ingress, although equally resistant to moisture outflow. With a mean stone-water contact angle slightly over $70^{\circ}$, the stone proved to be naturally water repellent.

The limestone used to build the former Hospital of Maudes sourced from Morata de Tajuña and the Colmenar de Oreja limestone both exhibited similar petrophysical performances (Table 4). Their ultrasonic wave velocity, real and bulk density, open porosity and vacuum saturation absorption values were all very similar. Both stones could be classified as high quality materials, apt for use in structural members and highly resistant to aggressive agents and time-induced wear.

The prevalent, albeit ephemeral, use of Morata de Tajuña limestone from regional quarries as a building material in the early twentieth century in the city of Madrid, where it was scarcely known before that time, adds value to the use of Cornicabra quarry material.

\section{Introducción}

El estudio sobre la procedencia y la calidad de la piedra tradicional (o de cantería) empleada en el patrimonio construido incluye diversos aspectos. Por la propia definición de piedra tradicional -material por lo general de alta resistencia mecánica que tras un proceso de elaboración puede ser utilizado como elemento constructivo, normalmente exento de pulido, conservando su composición y textura originales (Echevarría and García, 1996), que suele proceder de canteras y/o afloramientos cercanos (Varas et al., 2010; Fort et al., 2013, Pereira and Baltuille, 2014) y que participa en la imagen de los pueblos y ciudades que configura (Pérez-Monserrat et al., 2013)-, estos estudios remiten a los sustratos geológicos próximos y a su actividad extractiva. Por un lado, la consulta de archivos y fuentes documentales permiten en numerosas ocasiones contextualizar históricamente las explotaciones, conociendo algunos de los factores que condicionaron la selección y el uso de una piedra en una época determinada (Pérez-Monserrat et al., 2017). Por otro, también la consulta de los estudios geológicos previamente realizados resulta esencial, pues aportan información sobre la génesis y posteriores procesos acontecidos (hidrotermales, diagenéticos...), aspectos que otorgan a la piedra unas pro- piedades específicas. Los caracteres petrográficos, principalmente la composición y la textura, condicionan las propiedades petrofísicas que presenta la piedra, expresadas mediante una serie de parámetros que cuantifican su comportamiento (García del Cura et al., 1996; Esbert et al., 2008), permitiendo conocer su calidad para desempeñar una determinada función como material de construcción (Ordóñez et al., 1996).

A partir del estudio de la procedencia y de la calidad de la piedra caliza empleada en la construcción del Antiguo Hospital de Jornaleros de Maudes (PérezMonserrat, 2015), son dos los principales objetivos que se plantean en el presente trabajo. Por un lado, se abordan los principales motivos que favorecieron el empleo como piedra de cantería en la capital a principios del siglo XX de la caliza explotada en las canteras de Morata de Tajuña, frente a la presumible selección de la prestigiosa caliza de Colmenar de Oreja. Por otro, mediante su caracterización petrológica (petrográfica y petrofísica) y en base a los estudios geológicos consultados, se analiza la calidad de esta piedra como material de construcción, estableciendo también su comparativa con la caliza de Colmenar de Oreja. Este tipo de estudios refleja que cuando se aborda la procedencia y la calidad de la piedra tradicional empleada en el patrimonio construido, todos 
los factores considerados son importantes, pues en su conjunto condicionaron tanto su selección en una época concreta como su utilización para desempeñar una determinada función constructiva.

\section{Una caliza para el Hospital de Jornaleros de Antonio Palacios}

El Antiguo Hospital de Jornaleros de Maudes fue construido por el insigne arquitecto gallego Antonio Palacios Ramilo (1974-1945) entre los años 1909 y 1916 en la zona norte de Madrid, junto al antiguo Paseo de Ronda, hoy la calle Raimundo Fernández Villaverde, y muy cerca de la actual Glorieta de Cuatro Caminos. En la ideología y obra de Palacios, cuya familia era propietaria de unas canteras de granito en O Porriño (Pontevedra), cabe destacar la búsqueda del sentido de la naturaleza de los materiales, la utilización de sus propiedades intrínsecas para otorgar textura a las fachadas y el empleo de los materiales locales como apuesta por la identidad de los lugares donde construía (González-Amezqueta, 1967). EI inmueble, constituido principalmente por cuatro naves dispuestas en diagonal en torno a un patio central, se levanta en la época moderna, en el pleno apogeo del hierro y el cristal, de la prefabricación y de la estandarización de los proyectos, resultando ciertamente tradicional en cuanto a su planteamiento constructivo y material pétreo empleado se refiere. De este modo, los muros del Antiguo Hospital se corresponden con una fábrica mixta a dos caras, siendo la exterior de cantería en una piedra caliza correspondiente a material tradicional de la región, procedente de las canteras de Cornicabra ubicadas en el paraje del Valhondo de Morata de Tajuña, como se ha indicado (López-Urrutia 1926). Las fachadas están conformadas por bloques de morfologías y tamaños muy diversos, variabilidad que responde en gran medida a la necesidad de amoldar el despiece de los paramentos a la potencia de los bancos en las canteras, para conseguir el máximo aprovechamiento de la piedra. El edificio estuvo abandonado durante los años 19701984 y ha sido objeto de dos importantes intervenciones, en 1984-1986 y 2006-2008. Desde 1979 es Monumento Histórico Artístico y actualmente alberga la sede de diversas Consejerías de la Comunidad de Madrid.

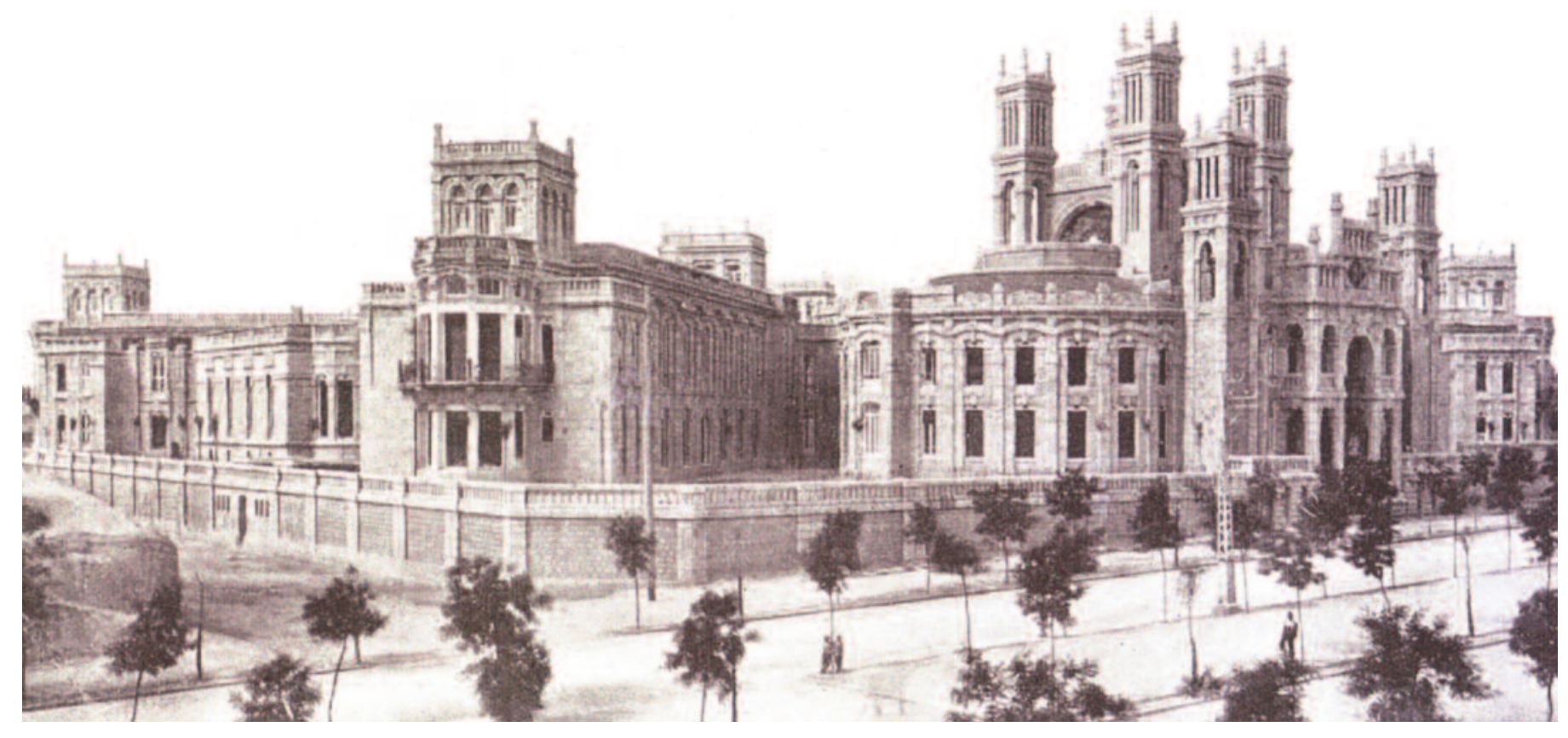

Figura 1. Hospital de Jornaleros de Maudes, construido entre 1908-1916 por el arquitecto gallego Antonio Palacios con piedra caliza procedente de Morata de Tajuña (Comunidad de Madrid). Cortesía del Centro Documentación Especializada, Consejería Medio Ambiente y Ordenación del Territorio (Comunidad de Madrid).

Figure 1. Former Maudes Workers Hospital, built with limestone from Morata de Tajuña, (Comunity of Madrid), by architect Antonio Palacios in 1908-1916. Courtesy of the Specialised Documents Centre, Madrid Regional Department of Environmental Affairs and Land Management. 


\section{Marco geológico y cantería en el sureste de la región}

Uno de los principales dominios que constituyen el sustrato geológico de la Comunidad de Madrid es la Cuenca de Madrid, donde tradicionalmente han existido numerosas canteras en las que se han explotado los carbonatos fluviolacustres del Mioceno Superior. En ninguna parte se extraía una piedra tan buena para configurar elementos constructivos como en las inmediaciones de Colmenar de Oreja (HernándezPacheco, 1926), cuya belleza se realzaba en superficies desbastadas y/o pulidas. Debido a su calidad, pureza y blancura, esta localidad fue sin duda la más significativa y concentró las más prestigiosas explotaciones, proporcionando el municipio la denominación de piedra de Colmenar (Madoz, 1847; Martín, 1994; Menduiña and Fort, 2005). Así, se ha venido empleando el término genérico piedra de Colmenar para referirse a calizas miocenas explotadas en zonas próximas, como Cuesta Zulema, Los Santos de la Humosa, Morata de Tajuña, Nuevo Baztán o Campo Real (Puche and Mazadiego, 1999; Martínez, 2000).

\section{Los carbonatos fluviolacustres de la Unidad Superior Miocena de la cuenca de Madrid}

La Cuenca de Madrid forma parte de la depresión terciaria del Tajo y su relleno sedimentario tuvo lugar durante el Terciario y el Cuaternario (figura 2a, tomada de Sanz-Montero et al., 2003). En el primer tercio del siglo XX destacan, entre otros, los trabajos de Hernández-Pacheco (1923 y 1926) y Royo (1929). A partir de los años sesenta, se profundiza en sus aspectos sedimentológicos (Quirantes, 1968; Riba et al., 1969); en los años 80 y 90, Alonso-Zarza et al. (1992), Calvo et al. (1989), García del Cura et al. (1991, 1994), Megías et al. (1983), Ordóñez and García del Cura (1983) y Sanz-Montero et al. (1995) aportan síntesis sedimentológicas y paleoclimáticas del relleno terciario de la cuenca.

El relleno de la cuenca, constituido por capas subhorizontales en el que se identifican tres unidades fundamentales, tuvo lugar principalmente durante el Mioceno. Estas tres unidades ya son referidas por Casiano de Prado en 1864, que señala una división inferior con areniscas y conglomerados, otra media con arcillas y yesos, y otra superior con calizas. Esta división superior -Unidad Superior Miocena- está formada por las unidades fluvio-lacustres terminales (Megías et al., 1983), que representan el relleno final de la cuenca. En esta unidad se diferencia un nivel inferior detrítico y un nivel superior carbonático, constituido por los carbonatos fluviolacustres del
Mioceno Superior o la serie carbonática de la Unidad Superior.

La Unidad Superior Miocena está muy ligada con los sistemas fluviales y refleja un cambio en el régimen general de la cuenca hacia un contexto exorreico con aguas cada vez más diluidas, evolucionando los sistemas lacustres de evaporíticos, correspondientes a la Unidad Intermedia, a carbonatados (Calvo et al., 1989), que se corresponden propiamente con dicha Unidad Superior. La heterogeneidad litológica de los márgenes que encuadran la cuenca así como su evolución paleoclimática, fueron los principales factores que condicionaron la formación del complejo mosaico de sistemas fluviolacustres en donde se depositó la serie carbonática miocena (Calvo et al., 1989). Este mosaico correspondía a lagos someros no muy extensos y de agua dulce interconectados por los ríos (Sanz-Montero et al., 2003), donde se desarrollaron las diferentes litofacies presentes en la serie (figura 2b, tomada de SanzMontero et al., 2003). Posteriormente al depósito de estos carbonatos, se suceden eventos sedimentarios, kársticos y erosivos durante el Plioceno, por exposición subaérea de las calizas al desecarse parcialmente la cuenca (Sanz-Montero et al., 2003).

Casiano de Prado (1864) describe las variedades que muestra esta serie y, por el contenido en fósiles dulceacuícolas, ya asociaba la serie con carbonatos de agua dulce. En el primer tercio del siglo XX se atribuía su génesis a un ambiente lacustre y palustre (Fernández-Navarro and Carandell, 1914; HernándezPacheco, 1923; Royo 1929). Castells and de la Concha (1951) identifican calizas tobáceas y cavernosas, y Riba et al. (1969) consideran facies oncolíticas y tobáceas. San José (1975) indica que la serie presenta numerosas variaciones locales en desarrollo y facies, masas de calizas lacustres, grises o blancas, compactas y duras, calizas tobáceas con abundantes tallos de plantas, y alternancia de calizas margosas, margas compactas y margas rojizas arenosas con cantos.

Bustillo (1980) identifica en estos carbonatos calizas homogéneas de ambiente subacuático tranquilo (micritas, microesparitas o pseudoesparitas), grumelares (con textura grumelar debido a gravels micríticos), lacustres, fosilíferas de zonas pantanosas con desecación (biomicritas con algas, gasterópodos y ostrácodos), brechoideas de ambiente palustre, oncolíticas y tobáceas de ambiente litoral y lacustre (con facies estromatolítica y de musgos), y karstificadas de ambiente pantanoso.

Ordóñez and García del Cura (1983) consideran que la serie también presenta facies de medios fluviales de baja energía, existiendo episodios de facies tobáceas, estromatolíticas y oncolíticas que alternan 
con facies palustres de estancamiento. Calvo et al. (1989) identifican facies tobáceas, oncolítitas, micritas fosiliferas y biomicritas, correspondiendo el amplio desarrollo de las facies tobáceas y oncolíticas a un episodio de importante fitoproducción. García del Cura et al. (1994) señalan que la variabilidad de facies de la serie, con carbonatos biogénicos en facies oncolíticas, estromatolíticas y tobáceas, calizas micríticas folislíferas con gasterópodos, ostrácodos y charáceas, es propia de ambientes fluviolacustres.

Debido principalmente a la gran variedad de facies existentes en los ambientes fluviolacustres y a los abundantes cambios laterales que presenta la serie carbonática del Mioceno Superior, facies de un determinado tipo suelen presentar rasgos característicos de otra (San José, 1975; Bustillo, 1980; García del Cura et al., 1994).

\section{Los carbonatos fluviolacustres de la Unidad Superior Miocena como piedra tradicional}

De Prado (1864) señala que donde el terreno no había sido erosionado, la horizontalidad del relleno superior de la cuenca daba lugar a extensas llanuras, que originaron el páramo castellano, de ahí que estos carbonatos se hayan denominado tradicionalmente calizas de los páramos (Hernández-Pacheco, 1945). Regionalmente, por calizas de los páramos se entiende el nivel superior de calizas lacustres que, debido a su resistencia a la erosión, constituyen la superficie de los páramos típicos que coronan las cubetas terciarias continentales del Duero, Ebro y Tajo. Estratigráficamente esta denominación resulta poco rigurosa, puesto que la superficie de los páramos no representa un nivel de depósito sino una superficie de erosión (Schwenzner, 1936), siendo más preciso hablar de los carbonatos lacustres de la Unidad Superior Miocena (Calvo et al., 1989) o de la serie carbonática de la Unidad Superior (García del Cura et al., 1994).

Los espesores de la serie decrecen de Norte a Sur, presentando en el eje del sinclinal de Morata de Tajuña las máximas potencias (San José, 1975) y concentrándose las principales explotaciones de estos carbonatos en el afloramiento que se extiende aproximadamente entre Colmenar de Oreja, Villar del Olmo y Arganda (figura 3). El río Tajuña divide en dos el afloramiento, resultando el páramo de ChinchónColmenar de Oreja entre los ríos Tajuña y Tajo, y el páramo de Arganda-Morata deTajuña en el interfluvio Jarama-Tajuña (García del Cura et al., 1994).

La caliza de Colmenar de Oreja es una piedra de cantería tradicionalmente muy utilizada en el patri-

monio construido de la Comunidad de Madrid (De Prado, 1864; Hernández-Pacheco, 1926 y 1945; GarcíaFernández, 1948; Dapena et al., 1989; García de Miguel et al., 1992; Martín, 1994; Fort et al., 1996a, 2013, 2015; Menduiña and Fort, 2005). La caliza procedente del páramo de Morata de Tajuña ha sido empleada también como piedra de cantería y es conocida la calidad ornamental del travertino de
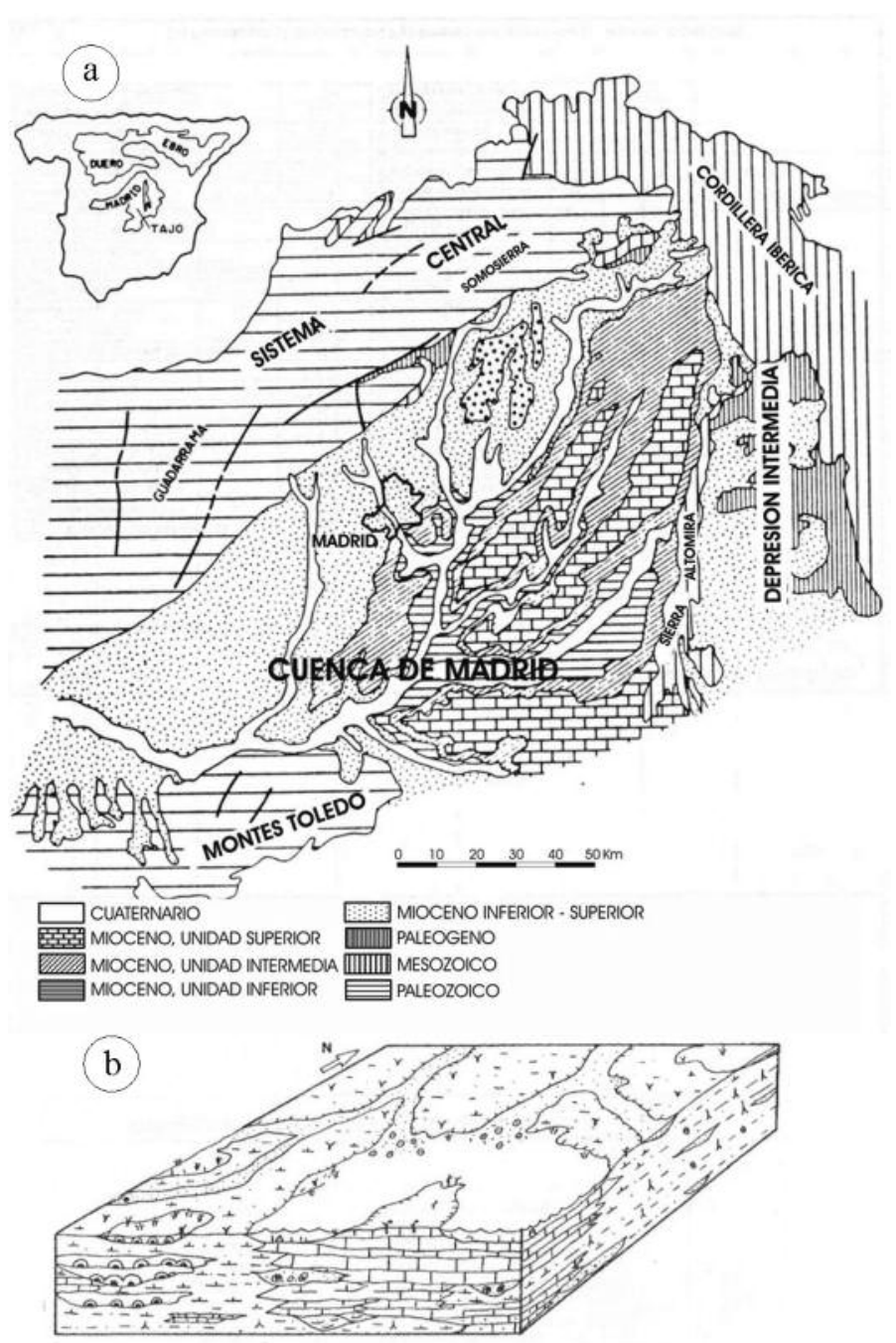

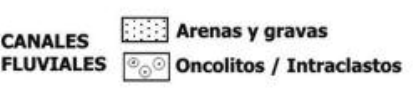

LLANURA DE INUNDACIÓN
Limos y lutitas

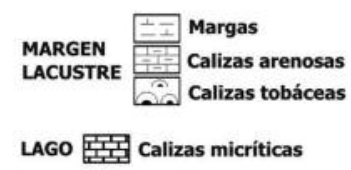

Figura 2. La cuenca terciaria continental del Tajo (tomado de SanzMontero et al., 2003). A) Mapa geológico simplificado de la cuenca y de sus márgenes. B) Modelo sedimentario de la Unidad Superior Miocena.

Figure 2. Madrids basin (from Sanz-Montero et al., 2003). A) Simplified geological map of the basin and its margins. B) Sedimentary model for the Late Miocene Unit. 
Morata (García del Cura et al., 1994), si bien el beneficio de esta caliza ha sido principalmente para la obtención de cal en la industria del cemento (Puche and Mazadiego, 1999).

En la ciudad de Madrid, la caliza de Colmenar de Oreja se ha empleado en construcciones tan emblemáticas como el Palacio Real (1738-1755), la Puerta de Alcalá (1769-1778) o la Casa de Velázquez (1923-1935). La caliza de Morata de Tajuña fue utilizada en el Antiguo Hospital de Jornaleros de Maudes (LópezUrrutia, 1926) o para la construcción de la primera línea del Metropolitano de la capital (De la Torre and Paris, 1986), inaugurada en 1919.

Hernández-Pacheco (1926) hace referencia a los primeros ensayos petrofísicos en la caliza de Colmenar de Oreja, señalando Dapena et al. (1989) y Fort et al. (1996a, 2011, 2013 y 2015) su buena calidad y durabilidad. De este modo, recientemente, la piedra de Colmenar ha sido propuesta como candidata para ser nominada como Recurso Pétreo del Patrimonio Mundial (Fort et al., 2015), resultando además una piedra de especial belleza, debido a su blancura y ausencia de impurezas.

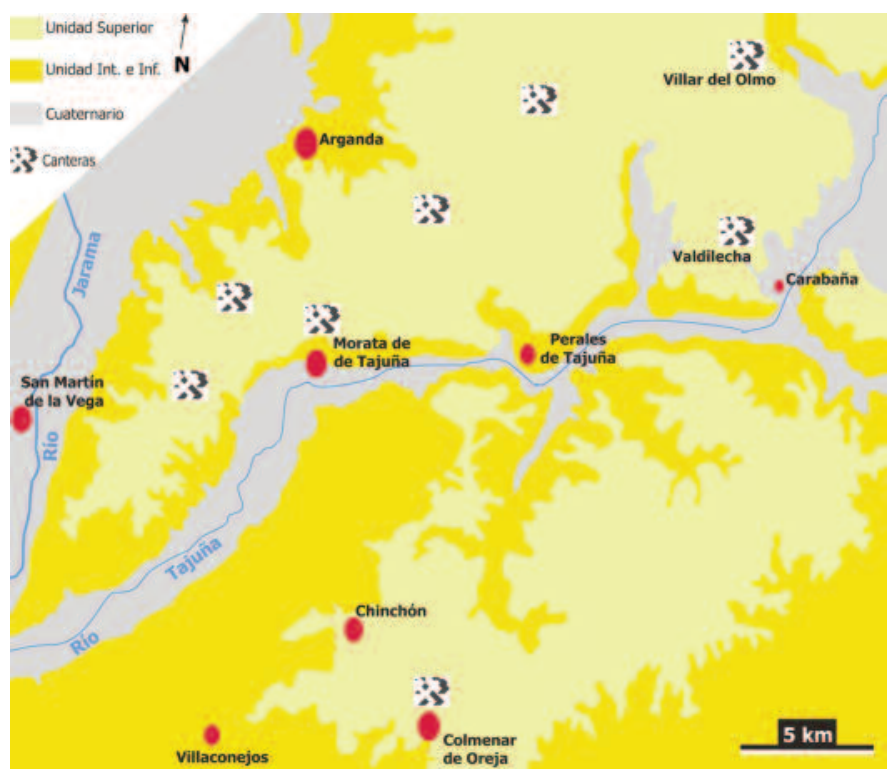

Figura 3. Mapa geológico simplificado de los páramos de Chinchón-Colmenar de Oreja y de Arganda-Morata de Tajuña y localización de las principales canteras donde los carbonatos fluviolacustres de la Unidad Superior Miocena son y/o han sido explotados (a partir de García del Cura et al., 1999).

Figure 3. Simplified geological map of the Chinchón-Colmenar de Oreja and Arganda-Morata de Tajuña high moors and location of the major sites where the Late Miocene Unit fluvial-lacustrine carbonates are or have been quarried (from García del Cura et al., 1999).

\section{Metodología}

\section{Estudios abordados y toma de muestras}

En primer lugar, se presenta la evolución histórica especialmente a finales del siglo XIX y principios del XX- de la explotación de caliza en las canteras de Colmenar de Oreja y de Morata de Tajuña así como de las vías de comunicación existentes por entonces y que conectaban ambas localidades con la ciudad de Madrid. Esta contextualización histórica ha permitido localizar en las canteras de Morata deTajuña al menos un frente de explotación antiguo.

En segundo lugar, se describe estratigráficamente este frente de cantera histórico y se comparan los caracteres petrográficos -textura y composición- de la caliza tomada del afloramiento y de las fachadas del Antiguo Hospital de Maudes. La procedencia señalada en las fuentes documentales debe confirmarse, pues en ocasiones puede variar debido a causas que no quedan recogidas en los documentos consultados, resultando muy adecuado este tipo de estudios comparativos (Gomez-Heras and Fort, 2004; Pereira and Baltuille, 2014). Posteriormente, se efectúa la caracterización petrofísica de la caliza del inmueble, mediante técnicas de caracterización no destructivas (TND) o mínimamente destructivas y la realización de ensayos normalizados.

Se ha tomado una muestra de cada una de las facies reconocidas en los tres tramos definidos en el frente de cantera. En la fachada sur de la nave noroeste del edificio se han extraído 4 testigos (denominados $A, B, C$ y $D)$ de $6 \mathrm{~cm}( \pm 1 \mathrm{~mm})$ de diámetro y 13$17 \mathrm{~cm}$ de longitud de un sillar del zócalo. Los sillares muestreados han sido posteriormente reintegrados mediante su relleno con árido calizo de diversas granulometrías y su sellado con mortero de restauración.

\section{Técnicas y ensayos de caracterización}

La caracterización petrográfica se ha realizado según la normativa UNE-EN 12407:2007, atendiendo a los caracteres texturales y composicionales tanto en la descripción macroscópica de las muestras tomadas como en el análisis petrográfico de las láminas delgadas (30 $\mathrm{m}$ de espesor), confeccionadas, que han sido teñidas parcialmente según método propuesto por Friedman (1959). Se ha empleado el microscopio de polarización Olympus BX51 con cámara digital acoplada Olympus DP12.

Para la caracterización petrofísica (tabla 1), únicamente efectuada en la caliza de las fachadas del 
Pérez-Monserrat, E. M., et al., 2017. La caliza de Morata de Tajuña, Comunidad de Madrid:... Boletín Geológico y Minero, 128 (4): $963-988$

inmueble, se han seleccionado algunos de los parámetros que caracterizan el aspecto y comportamiento mecánico de la piedra (color, densidad, dureza, compacidad o anisotropía) así como los que definen su respuesta a la acción del agua (porosidad, saturación o permeabilidad).

Espectrofotometría, determinación de parámetros cromáticos: se han considerado la luminosidad $\left(L^{*}\right)$, con valores comprendidos entre 0 (negro puro) y 100 (blanco puro), las coordenadas cromáticas $a^{*} y b^{*}$, que representan el grado de saturación hacia el color verde $\left(-a^{*}\right)$ y rojo $\left(+a^{*}\right)$ y hacia el azul $\left(-b^{*}\right)$ y el amarillo $\left(+b^{*}\right)$, y la croma $\left(C^{*}\right)$ o saturación del color, que determina el grado de separación entre el color puro y el color gris o acromático, calculada en base a la fórmula $C^{*}=\left[\left(a^{*}\right)^{2}+\left(b^{*}\right)^{2}\right]^{1 / 2}(C I E, 1978)$. También se han considerado los índices de blanco y de amarillo (ASTM E313:2010). Se ha empleando el espectrofotómetro CM-2002 de Minolta y el software Colour Data CM-1, seleccionando el iluminante estándar de la CIE D65, un ángulo de visión o ángulo del observador de $10^{\circ}$ y un patrón blanco de referencia

Esclerometría, determinación de la dureza superficial (ASTM D5873:2000): el cálculo de este parámetro resulta especialmente importante en materiales pétreos destinados a configurar elementos constructivos (Yasar and Erdogan, 2004). Este ha sido el único ensayo que se ha efectuado en el edificio, directamente sobre la superficie del sillar muestreado. Se ha empleado el esclerómetro portátil Schmidt Type $\mathrm{N}$ de Proceq, midiéndose la energía de rebote como una medida de resistencia a la compresión $\left(\mathrm{kg} / \mathrm{cm}^{2}\right.$ ó $\mathrm{N} / \mathrm{mm}^{2}$ ) o por el índice de rebote $\mathrm{R}(10-100)$.

Velocidad de propagación de las ondas ultrasónicas (EN 14579:2005): la determinación de este parámetro ( $\mathrm{Vp}$, en $\mathrm{m} / \mathrm{s}$ ) se basa en la propagación de los ultrasonidos, dependiendo el tiempo de propagación del impulso ultrasónico principalmente de la densidad del material y de sus heterogeneidades (Álvarez de Buergo and González-Limón, 1994). Cuanto más elevada es esta velocidad, mejor es la calidad del material en cuanto a su resistencia a los esfuerzos de compresión principalmente. Se ha empleado el equipo de medida Pundit CNS Electronics. Se han determinado además los índices de anisotropía total $(\Delta \mathrm{M} \%$ $\left.=\left[1-\left(2 V p_{\text {min }} /\left(V p_{\text {media }}+V p_{\text {max }}\right)\right)\right] \times 100\right)$ y relativa $(\Delta m \%$ $\left.=\left[\left(2 \times\left(2 V p_{\max }-V p_{\text {media }}\right) /\left(V p_{\text {media }}+V p_{\text {max }}\right)\right)\right] \times 100\right)$ establecidos por Guydader and Denis (1986). El conocimiento de la anisotropía tiene una influencia directa a la hora de colocar los elementos constructivos en obra (Fort et al., 2011); valores bajos en los índices de anisotropía revelan igualmente una elevada calidad del material y si además resultan similares el material no presenta direcciones preferentes (Zezza, 1990).
Ensayo de saturación, determinación de la densidad (real y aparente), de la porosidad accesible al agua y del porcentaje de agua de saturación (RILEM P25 1980): el valor de la densidad aparente siempre es inferior al de la densidad real y la diferencia obtenida entre ambos valores permite una aproximación al sistema poroso del material, puesto que cuanto más similares sean menor es su porosidad accesible al agua (Fort, 1996b; UNE-EN 1936:2007). El porcentaje de agua de saturación se define como la cantidad máxima de agua que un material es capaz de absorber por saturación en condiciones de vacío y está directamente relacionado con la porosidad accesible al agua.

Porosimetría por Intrusión de Mercurio: para cada presión ejercida, el mercurio penetra en un radio de poro determinado, estableciéndose la porosidad del material para ese tamaño mediante la cuantificación del volumen de mercurio intruido. A partir de la relación entre la presión ejercida y el tamaño de los poros, la cantidad de mercurio introducido a distintas presiones permite confeccionar curvas de distribución porosimétrica (Rodríguez-Navarro and Sebastián, 1994; Fort, 1996b). En términos generales, una mayor variabilidad en los intervalos de tamaño (curvas polimodales) señala que más diferentes son los tamaños de los poros y, por tanto, mayor susceptibilidad tendrá el material a degradarse por la acción del agua, pues mayor es la cantidad de agua que retiene en su interior y más fácil es su movilidad (Fort, 1996c). Por otro lado, cuanto menor sea el tamaño de poros, menor capacidad de movimiento tendrá el agua en el interior del material, aspecto que otorga mayor durabilidad al material. Se ha empleado el equipo Autopore IV 9500 de Micromeritics, que permite la medida de tamaño de poros comprendidos entre 0,001 y 400 m mediante la intrusión de mercurio a presiones de 15 a 60.000 psi, estableciéndose el umbral de diámetro de poro entre micro y macroporosidad en $5 \mathrm{~m}$ (Rodríguez-Navarro and Sebastián, 1994; Fort et al., 2011).

Permeabilidad al vapor de agua (NORMAL 21/85): el ensayo mide la cantidad de vapor de agua que es capaz de atravesar un material para un intervalo de tiempo dado cuando se establece un gradiente de presión entre dos superficies paralelas, definida por el coeficiente de permeabilidad, medido en $\mathrm{g} / \mathrm{m}^{2} \cdot 24 \mathrm{~h}$ $\mathrm{mmHg}$.

Determinación del ángulo de contacto (UNE-EN 15802:2010): el ángulo de contacto, medido en grados sexagesimales $\left({ }^{\circ}\right)$, que se forma entre una gota de agua sobre una superficie sólida está relacionado con el carácter hidrófobo de dicha superficie. Cuanto mayor sea el ángulo de contacto entre la superficie y el agua, mayor será la capacidad hidrofugante de la 
Pérez-Monserrat, E. M., et al., 2017. La caliza de Morata de Tajuña, Comunidad de Madrid:... Boletín Geológico y Minero, 128 (4): $963-988$

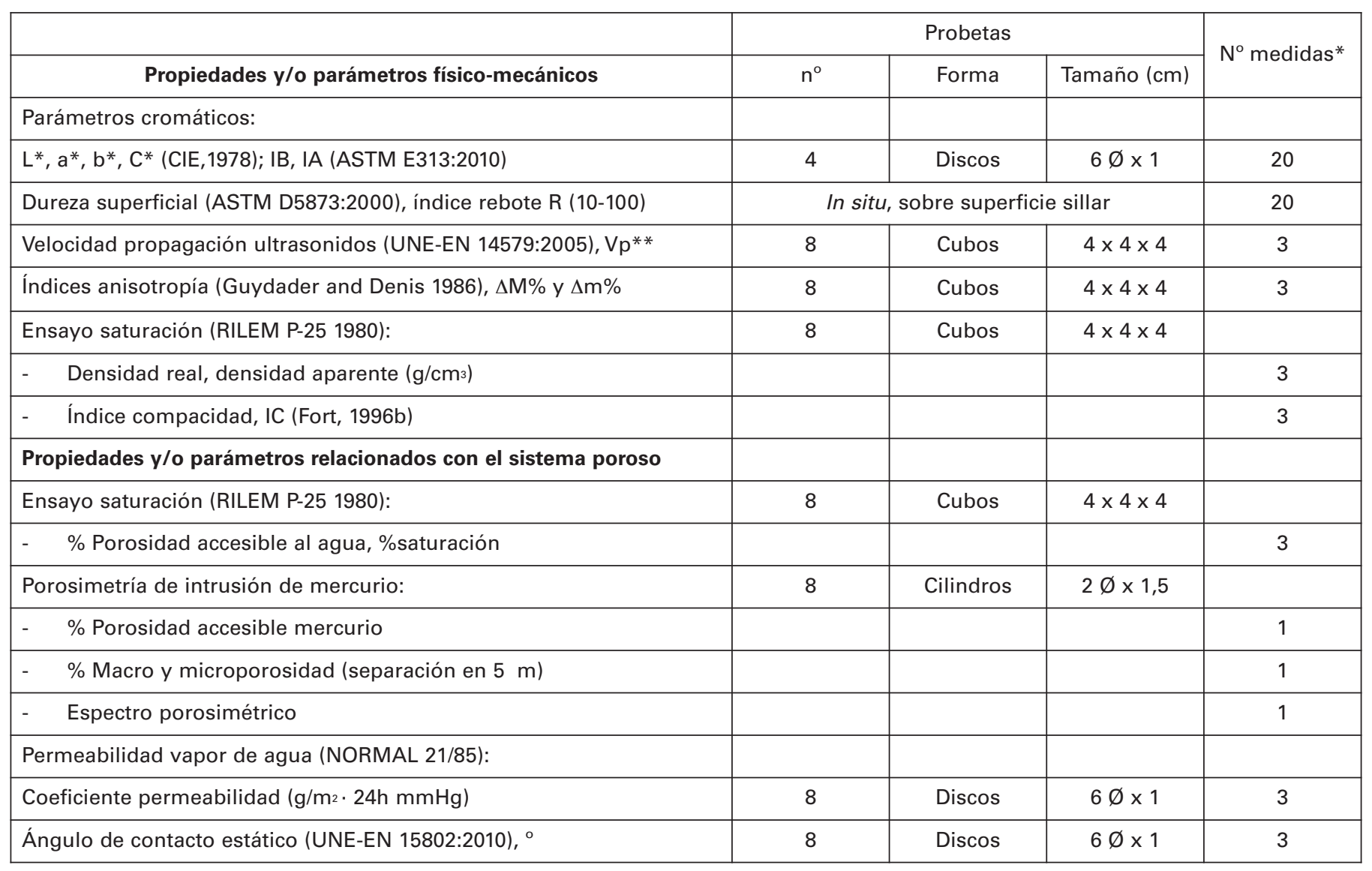

*número de medidas efectuadas en cada probeta.

**Vp determinada en las tres direcciones del espacio de las probetas cúbicas.

Tabla 1. Caracterización petrofísica de la caliza empleada en la construcción del Antiguo Hospital, procedente de Morata de Tajuña.

Table 1. Petrophysical characterisation of the Morata de Tajuña limestone used to build the former Maudes Workers' Hospital.

superficie y, por tanto, mayor resistencia presentará a la entrada de agua (Fort, 1996c). Cuanto más próximo a $90^{\circ}$ sea el valor del ángulo de contacto, mayor capacidad hidrofugante de forma natural presentará la superficie. Se ha empleado el analizador de ángulo de contacto estático modelo Phoenix 300 de SEO (Surface Elctro Optics).

\section{Resultados}

\section{Contextualización histórica}

Explotaciones de caliza en las canteras de Colmenar de Oreja y de Morata de Tajuña

Páramo Chinchón-Colmenar de Oreja: la caliza de Colmenar de Oreja

En el siglo XVI ya existía en Colmenar de Oreja una cantera a cielo abierto, en Navarredonda, saliendo por el Norte del pueblo (Martín, 1994). El cuaderno de familias del año 1751 indicaba que "el floreciente sector de la cantería que se ocupa en sacar de las entrañas de la tierra, a corta distancia del pueblo, la célebre piedra caliza o blanca de Colmenar, con la que se viene labrando en buena parte el Palacio Real de Madrid" (Hurtado, 1991). Los catastros del Marqués de la Ensenada (1752) y del cardenal Lorenzana (hacia $1780)$ hacen referencia, respectivamente, a las canteras existentes en el término municipal de Colmenar de Oreja y a las magníficas cualidades de la caliza extraída en las mismas (Jiménez de Gregorio, 1978).

Madoz (1848) indica que la segunda mitad del siglo XVIII fue el punto álgido para esta caliza y, cuando se refiere en su diccionario a Colmenar de Oreja, señala que "a la parte del Norte se ven canteras de piedra blanca Ilamada de Colmenar, de donde se extrajo la que se necesitó para los palacios de Madrid y Aranjuez y otros muchos edificios. En el día hay 
Pérez-Monserrat, E. M., et al., 2017. La caliza de Morata de Tajuña, Comunidad de Madrid:... Boletín Geológico y Minero, 128 (4): $963-988$

poca extracción, estando limitada a algún baño y piedra para las tahonas de Madrid". De Prado (1864) señala que la mejor piedra de sillería de la provincia estaba en el terreno terciario de Colmenar de Oreja, y que los canteros de la localidad denominaban a cada capa con un nombre diferente, siendo la superior el cabezal y siguiéndole el banquillo, el sobre banco, el banco gordo, el banco de levante y el banco vidrioso. La piedra para ornamentos se extraía principalmente del cabezal y los bloques más grandes del banquillo, banco gordo y banco de levante. El Boletín de la Revista de Obras Públicas del año 1896 se refiere a la necesidad de diseñar y despiezar los muros según las dimensiones de los bancos en las canteras, para conseguir el máximo beneficio del material. Igualmente, indica el Boletín que uno de los problemas que presentaba la caliza de Colmenar de Oreja era que para alcanzar los bancos con la dimensión requerida, era necesarias importantes labores de desmonte. Tanto el arranque como el desaprovechamiento de este material encarecían el coste final de la caliza.

A finales del siglo XIX y comienzos del XX, las canteras que habían sido explotadas durante el XVIII estaban bastante abandonadas (Madoz, 1848; Martín, 1994; Sancho-Gaspar, 1996; Puche and Mazadiego, 1999). Más que al agotamiento de los frentes, dicho abandono respondía fundamentalmente al mayor coste que la caliza de Colmenar de Oreja presentaba frente a calizas procedentes de otros puntos de la región y del país, principalmente del Levante español (De Pablos, 1891; Fort et al., 2002; Varas et al., 2003). A partir de 1920, la Sociedad General de Cementos Pórtland efectuaba la extracción a cielo abierto con el empleo de explosivos (Hernández-Pacheco, 1926). El mismo autor indica que las explotaciones eran numerosas, de baja potencia y muy extensas. Señala también que desde tiempos romanos y hasta los años cincuenta, la caliza se explotaba en galerías subterráneas más o menos horizontales que perseguían la uniformidad y potencia de los bancos. Estas galerías eran sostenidas con pilares constituidos por las mismas capas de roca, dejando tragaluces que permitían aprovechar la luz del día. Aunque la horizontalidad de las capas facilitaba la extracción, el método de explotación entrañaba cierto peligro, pues el estéril alcanzaba incluso los $10 \mathrm{~m}$ y la falta de medios económicos condicionaba la ausencia de técnicas de desmonte. Se explotaba el banco de levante y se dejaba caer el banco gordo, que se arrastraba hacia el exterior para su posterior preparado y serrado. Luego se profundizaba en el banco de levante y se extraían de nuevo los bloques del techo (Dapena et al., 1989; Puche and Mazadiego, 1999).

Durante el primer tercio del siglo $\mathrm{XX}$ la explota- ción de caliza se mantuvo (Estadística Minera 19091930), pero a menor ritmo de producción (Puche and Mazadiego, 1999), empleándose en importantes construcciones de la capital, como en la casa Velázquez (1923-1935). Kindelan and Cantos (1946) indican la existencia en Colmenar de Oreja de zonas de arranque de caliza destinada al dimensionado de grandes piezas de cantería y para la producción de áridos. Hoy en día, la producción se centra en la obtención de bloques, áridos de machaqueo y roca ornamental.

Páramo Arganda-Morata de Tajuña: la caliza del Valhondo y las canteras de Cornicabra

Se han localizado escasas referencias sobre la utilización antiguamente de la caliza de Morata de Tajuña como elemento de cantería. Utanda (2004) hace referencia a la existencia de una cantera de piedra "azucarada" hacia 1826, y Muñoz de Torres (1993) señala que Morata es tierra de piedra caliza, destacando su importantísima tradición calera y yesera.

Cuando Madoz (1848) se refiere a Morata, especifica la existencia de una cantera de piedra "azucarada" bastante limpia y blanca, de canteras de piedra tosca localizadas en los cerros del Norte de la localidad y de una piedra rica en yeso. El dato más antiguo localizado sobre la extracción de bloques de caliza se refiere a la construcción de la iglesia parroquial de Morata, finalizada en 1635, procediendo la caliza de una cantera ubicada en un cerro del municipio (De la Torre, 1999). De Diego (1891), indica que a finales del XIX se conservaban en dicha cantera piezas labradas de grandes dimensiones para la continuación de la iglesia. Fernández-Navarro (1907) señala que hacia el Norte de Morata, en una zona elevada, había una explotación de caliza terciaria, tobácea en gran parte y con escaso contenido fosilífero. En 1908, la Estadística Minera recoge una importante producción diaria de cal por la Sociedad de Cementos y Canteras de Valhondo (Puche and Mazadiego, 1999).

El Archivo Histórico Ferroviario del Museo del Ferrocarril de Madrid - Fundación de los Ferrocarriles Españoles dispone de un documento realizado por la Sociedad Explotadora de las Canteras de Cornicabra (ubicadas en el paraje del Valhondo, en el municipio de Morata de Tajuña) con motivo de la Exposición de Industrias Madrileñas celebrada en 1907. El documento (figura 4) aporta interesante información sobre la explotación en las canteras de Cornicabra a principios del siglo XX, así como sobre las excelentes propiedades de la piedra caliza que era allí extraída, que precisamente recibió un premio en dicha exposición.

En la figuras $4 b$ y $4 c$ puede observarse que la pro- 
ximidad de los bancos de caliza a la superficie minimizaba la eliminación del estéril, permitiendo además el máximo aprovechamiento del material. En la cantera, donde trabajaba un elevado número de operarios y parte de la caliza se calcinaba en el horno instalado junto al apeadero, la piedra era extraída y dimensionada de forma artesanal, principalmente mediante el empleo del pico. Las piezas de menor tamaño eran trasladadas con carretillas $y$, para los bloques extraídos en las zonas más elevadas de la cantera, se empleaban unas traviesas de madera que facilitaban su traslado.

También puede atenderse a la enorme ventaja que supuso, para el beneficio de la piedra caliza, la explanación de la zona anexa a las canteras y la construcción del apeadero. Los bloques, sin desbastar, se cargaban directamente en las vagonetas, que se acoplaban al ferrocarril. La operación era facilitada por la existencia de un muro bajo de mampostería, que impedía además el deslizamiento del terreno.

Puche and Mazadiego (1999) señalan que la Sociedad de Cementos y Canteras de Valhondo fue la primera en explotar la caliza de Morata para fabricar cemento, indicando la Estadística Minera de 1908 que producía casi 5500 kilos de cal al día. Mientras que esta Sociedad explotaba la caliza de las canteras del Valhondo principalmente para la fabricación de cal y cemento, la Sociedad Canteras de Cornicabra extraía la caliza de las canteras de Cornicabra fundamentalmente para su dimensionado como elemento de cantería. Los mismos autores, comentan que en 1915 la Estadística Minera recoge un acusado declive en la producción de las canteras madrileñas por la crisis en la construcción, existiendo en los años sucesivos explotación de caliza únicamente en Morata, Arganda y Campo Real.

En 1923 se instala en Vicálvaro la empresa Portland Valderribas S.A., trasladándose allí la producción de cal desde las canteras del Valhondo cuando la fábrica se pone en marcha en 1926. Vicálvaro contaba con importantes yacimientos de arcillas, y la empresa siguió explotando la caliza de los altos de Morata para la fabricación de cementos (Hernández-Pacheco, 1945; De la Torre and Paris, 1986). García-Fernández (1948) indica que, hacia finales de los años cuarenta, la caliza de Morata deTajuña competía para el dimensionado de elementos de cantería con la caliza de Colmenar de Oreja. Sin embargo, esto no actuaba en detrimento de ésta última, al ser la caliza que entonces se explotaba en Morata deTajuña de peor calidad y emplearse tan sólo cuando la demanda de caliza era muy alta, como ocurrió durante la construcción del Ministerio del Aire (1943-1958).

Aunque abandonado ya el frente de explotación,
Piedra caliza - Sociedad explotindora de las canteras de Cornicabra

En las laderas del término de Morata, divisorias de los rios Jarama y Tajuña, se halla el extenso e inagotabie macizo de piedra caliza que atraviesa la linea del ferrocaril de Madid a Coimenar, por el sitio denominado CORNICABRA del que toman nombre estas canteras

Aunque conocidas y reputadas desde tienpos remolos, impedia o dficultaba su explotacón la especial situación en la que se hal laban y carestia de su extracción y transporte, la que ha sido allanada y resulta por la actual Sociedad Explodadora construyendo al efecto en Cornicabra, kilometro núm 40 de la linea del ferrocarril del Tajufa, una Apartadero-Estación con toclas las condiciones y servicios necesarios a las exigencias del negocio, en forma tal, que no solo la piedra de mamposteria gruesa y sileria de cornentes dimensiones, sino que también los mas grandes bloques se traslacan desde su arranque a los vagones con gran faclicad escasisimo estuerzo e insignificante coste, lo cual facilita a esta Sociedad poder ceder o vender sus productos a los ventajosos precios que se fian o señalian en la presenta tarifa. que resultan más bajos y económicos que los que cotizan las procedencias de todas las demas canteras de piecras graniticas calizas o siliceas, a pesar de su inferioridad en belieza, calidad y resistencia en relación a las de Cornicabra Lo manifestado nos hace esperar que por estas razones y la mayor solicez de las cbras, los Sres. Arquitectos, Contratistas y Propietarios reemplazarán en cimentaciones los actuales matefiales, generalizando el empleo de este y a la vez podrán dar una mayor una mayor extensión a la silleria de las fachadas.

Como muestra, y para poder juzg ar los productos de estas Canteras, pueden verse, además de la instalación en la Exposición de industrias Macrileña en el Retiro, las columnas del Monumento a los Martires de la Patria en el parque del Deste, holel del St. Duque de Arevalo del Rey diversa s fachadas de casas de reciente construcción en las principales calles de esa Capital y la nueva estacion y edficios del Ferrocarnl del Tajufia
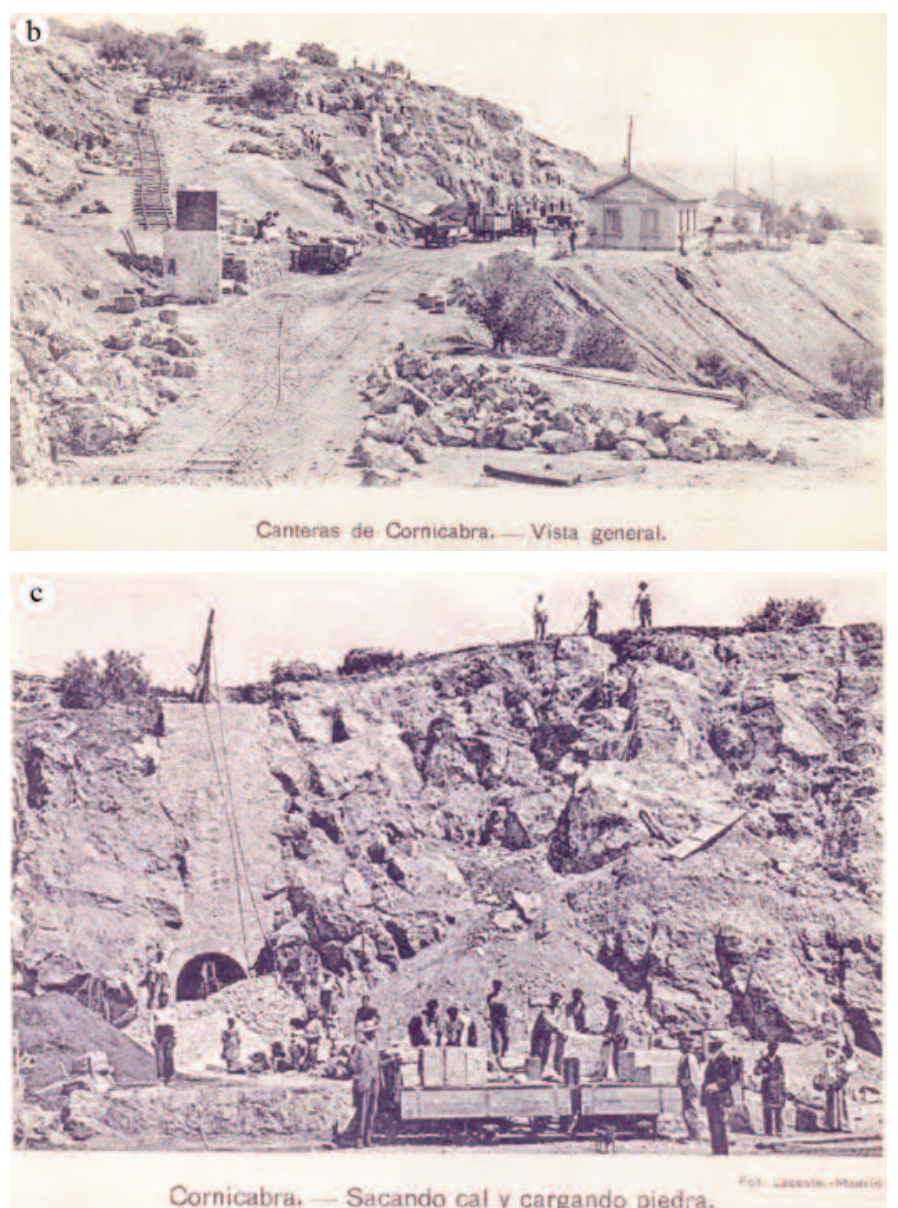

Figura 4. Documento realizado por la Sociedad Explotadora de las Canteras de Cornicabra, con motivo de la Exposición de Industrias Madrileñas de 1907. Cortesía del Archivo Histórico Ferroviario del Museo del Ferrocarril de Madrid - Fundación de los Ferrocarriles Españoles.

Figure 4. Document edited by the Sociedad Explotadora de las Canteras de Cornicabra (Cornicabra quarry company) on the occasion of the 1907 Madrid Industrial Fair. Courtesy of the Fundación de los Ferrocarriles Españoles, from the Historic Railway Archives custodied at its Madrid Railway Museum. 
hoy en día todavía pueden observarse vestigios de la actividad minera que antaño existió en la cantera, como lo que fue el horno calero y la casa del guarda, marcas dejadas por las herramientas utilizadas para el corte de los bloques, bancos que dejaron de explotarse, piezas pétreas labradas que no llegaron a trasladarse, así como el antiguo trazado ferroviario, hoy convertido en ruta verde (figura 5).

Vías de comunicación en el Sureste de la región durante los siglos $X I X$ y $X X$

En 1846, comenzaron las obras de la primera línea de ferrocarril de la provincia, inaugurada en 1851, que unió Madrid con Aranjuez. En 1856, se constituye la Compañía de Ferrocarriles de Madrid a Zaragoza y a Alicante (MZA). En 1858 se inaugura la primera línea de largo recorrido de España, que conectará las ciudades de Madrid y Alicante, y en 1860 ya existía la línea Madrid-Guadalajara, prolongada hasta Zaragoza en 1868 (González-Yanci, 1974). Los expe- dientes de la Compañía MZA del periodo comprendido entre los años 1880 y 1900, referentes al transporte ferroviario de la caliza de Colmenar de Oreja a Madrid, reflejan que el descenso acusado por entonces en la actividad de las canteras se debía principalmente a lo costosa que resultaba frente a las calizas procedentes del Levante español. Así, el ferrocarril de Alicante permitió la entrada en Madrid de piedras de construcción de otros lugares que resultaban económicamente más ventajosas que la caliza de Colmenar de Oreja (De Pablos, 1891; Fort et al., 2002; Varas et al., 2003).

A la dificultad que suponía la explotación de la caliza de Colmenar de Oreja mediante canteras subterráneas y al desmonte que era necesario efectuar para alcanzar los bancos con las dimensiones requeridas, debía añadirse el acarreo de la misma hasta Aranjuez, desde donde era trasladada por ferrocarril hasta Madrid. La llegada del tren a Colmenar de Oreja en 1903 reactivó las canteras y se abrieron nuevos frentes pues el transporte de la piedra a Madrid resultaba más barato, recuperando la caliza Colmenar de
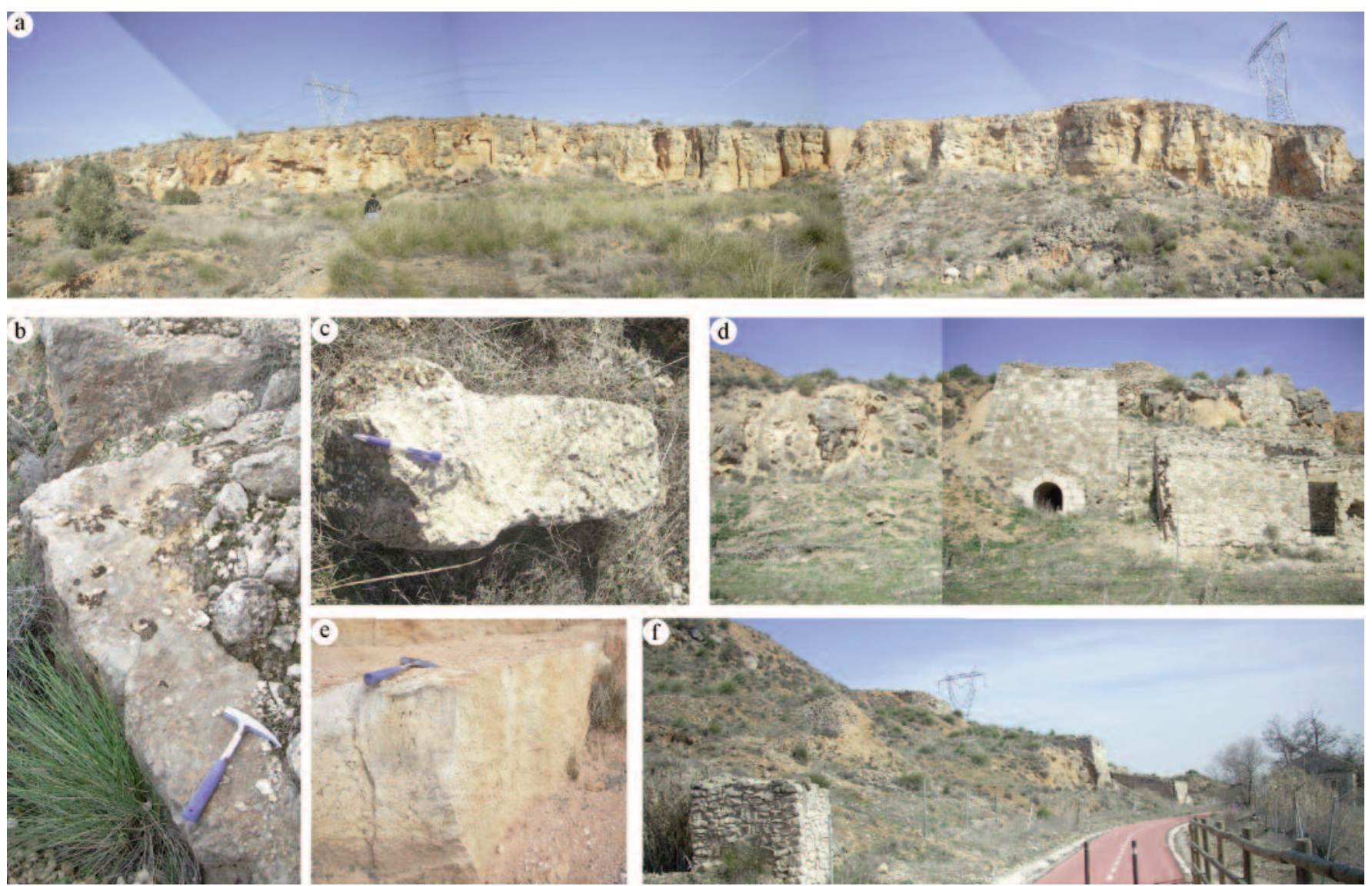

Figura 5. Estado actual de las canteras de Cornicabra en el paraje del Valhondo, Morata de Tajuña (Comunidad de Madrid).

Figure 5. Cornicabra quarries today in the Valhondo natural area, Morata de Tajuña (Comunity of Madrid). 
Oreja parte de su esplendor (García-Fernández, 1948). Indica el autor que el transporte de esta caliza mediante camiones también participó de su resurgimiento, destinándose para obras como en el Viaducto, el parque de las Vistillas, la plaza de Santa Ana o establecimientos del Retiro. De hecho, la estación ferroviaria de Colmenar de Oreja era una de las que más recaudaba y desde donde se trasladaba la piedra más importante de la localidad, denominada "marmolillos" (De la Torre and París, 1986).

Para el transporte de la caliza de Morata a Madrid, fue esencial el ferrocarril de Arganda o del Tajuña (figura 6 y tabla 2), que partía desde la estación del Niño Jesús, junto al parque del Retiro. En sus comienzos, sólo se planteó un servicio de mercancías entre Madrid y Vallecas para transportar materiales de construcción (González-Yanci, 1974). Las inauguraciones en la línea pudieron efectuarse cuando la compañía belga Caminos de Hierro compra el ferrocarril (Rodríguez, 2003).

En 1886 llega a Arganda y en 1901 a Morata, con una comisión especial para el reconocimiento de sus canteras de piedra. Las estaciones próximas a Morata eran El Alto -entre Arganda y Morata-, Cornicabra -en el paraje del Valhondo- y Morata -entre Morata y

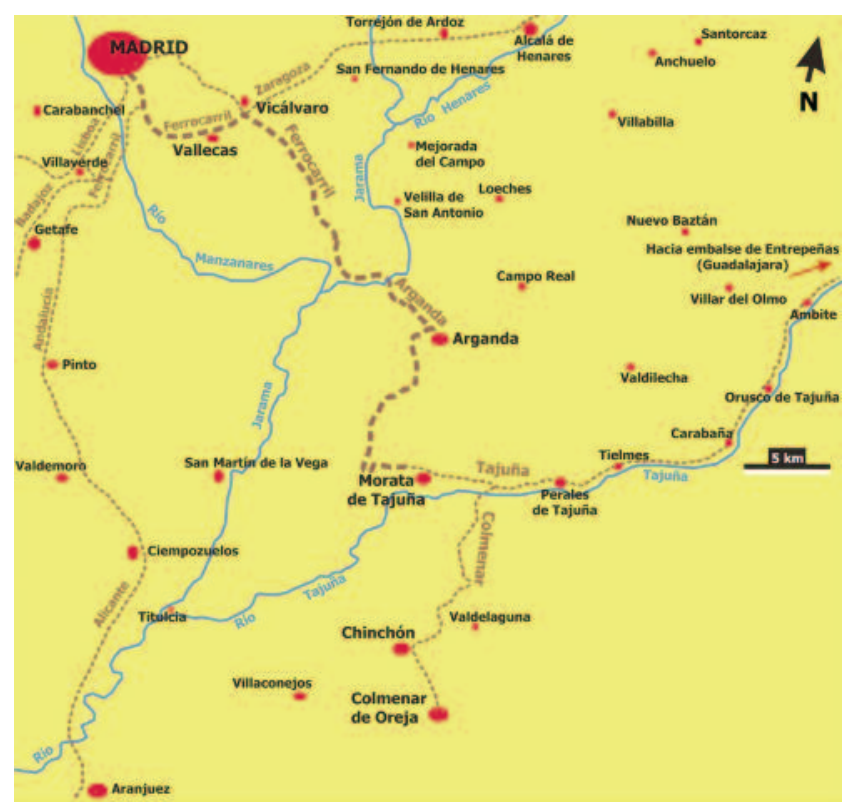

Figura 6. Mapa simplificado del Sureste de la provincia, mostrando el trazado ferroviario existente hacia 1925 (a partir de HernándezPacheco, 1926). La piedra caliza de Cornicabra era transportada por ferrocarril hasta la capital (línea discontinua gruesa).

Figure 6. Simplified map of south-eastern Comunity of Madrid province showing the railway network around 1925 (from HernándezPacheco, 1926). Cornicabra limestone was carried by rail to the capital city (thick dashed line).
Perales- (De la Torre and Paris, 1986). El proyecto de la construcción definitiva destinada a la casa del guarda y a la oficina de tráfico en el apeadero de Cornicabra, está fechado en 1905 (AGA, Sig. Caja 26/21860). En este documento, se indica que el proyecto para la construcción del apeadero se firma en 1904 y es aprobado a principios del siguiente año. Por tanto, si bien el ferrocarril llega a Morata en 1901, el apeadero de Cornicabra no será construido hasta 1905.

El ferrocarril también proyectaba articularse en Aranjuez, desde el ramal que llegaba hasta Colmenar de Oreja, con la línea Madrid-Alicante. En 1928 se alcanzó el máximo transporte de mercancías, principalmente por la carga de caliza para cal en el apeadero de Cornicabra. Sin embargo, la expansión del ferrocarril es paralizada tras la Guerra Civil, quedando definitivamente anulada en 1943 al construirse el embalse de Entrepeñas en Guadalajara (JiménezVega and Polo, 2001), bajo cuyas aguas yace la estación de Alocén (Rodríguez, 2003). En 1964, la empresa Portland Valderribas compra el tramo ferroviario entre las canteras de Cornicabra y su cementera de Vicálvaro, convirtiéndose el tren en un ferrocarril industrial que funcionó hasta 1998. La línea se cierra definitivamente en 1999, al constituir el tramo Vicálvaro-Arganda la recién inaugurada prolongación de la línea 9 del Metropolitano (De la Torre and Paris, 1986; Jiménez-Vega and Polo, 2001; Rodríguez, 2003).

\section{Caracterización de la caliza de las canteras de Cornicabra en Morata de Tajuña}

Descripción estratigráfica del frente de cantera y descripción macroscópica de la caliza de cantera y del edificio

Para acceder al frente de cantera histórico localizado, puede tomarse la carretera M-302 desde Morata de Tajuña dirección a San Martín de la Vega y pasado un mojón ubicado a la izquierda de la carretera que anuncia el paraje del Valhondo, a un kilómetro aproximadamente sale a la derecha un camino de tierra que llega hasta la antigua cantera de Cornicabra.

El frente de cantera histórico localizado presenta una orientación Noroeste-Sureste y la estratificación es horizontal (figura $5 \mathrm{a}$ ), buzando suavemente $\left(<10^{\circ}\right)$ hacia el Sureste. Este buzamiento se corresponde con la deformación tectónica regional que origina el sinclinal de Morata (San José, 1975). Las capas de caliza muestran intensa fracturación, la base de la serie está cubierta y hacia techo se observa el desarrollo de un perfil edáfico, identificándose también un depósito detrítico de coluvión cuaternario. Se observan estruc- 


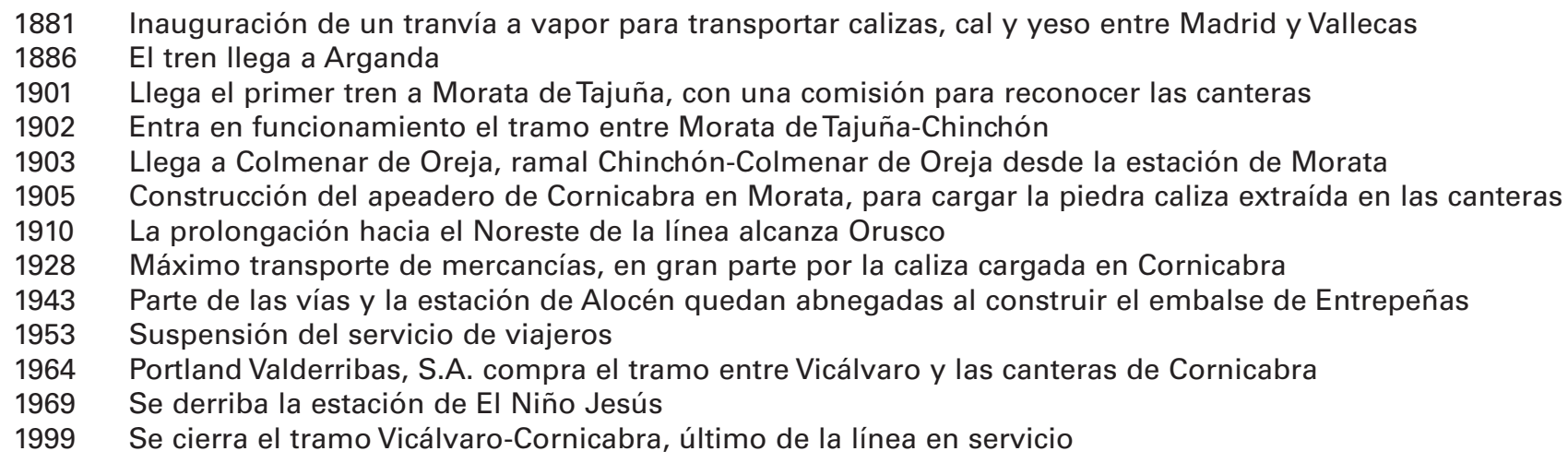

Tabla 2. Fechas significativas en la historia del ferrocarril del Tajuña para el transporte de la caliza de las canteras de Cornicabra (Morata de Tajuña).

Table 2. Timeline of significant dates in the history of the Tajuña railway used to carry limestone from the Cornicabra quarries at Morata de Tajuña to the city of Madrid.

turas de colapso, generadas posiblemente por eventos kársticos actuales, que han afectado sensiblemente al depósito cuaternario.

La serie, de $17 \mathrm{~m}$ de potencia, es estratocreciente y los planos de fracturación, generalmente perpendiculares a la estratificación, suelen encontrarse rellenos de abundante material arcilloso. La serie, constituida principalmente por calizas oncolíticas esparíticas y tobáceas, se ha dividido en tres tramos principales, constituidas a su vez por varios bancos (figura 7).

Tramo 1 - basal (3 metros de potencia):

1.1: banco de 1,2 $\mathrm{m}$ de potencia constituido por capas de calizas grisáceas oncolíticas con gravels y otros bioclastos -charáceas y gasterópodos- muy cementadas.

1.2: banco de $0,5 \mathrm{~m}$ de espesor de capas de calizas oncolíticas con intensa tonalidad gris, con bioclastos y gravels muy cementada. Hacia techo la facies muestra una tonalidad más clara y abundantes oncolitos y se observan cementos laminares de calcita similares a espeleotemas de tipo colada.

1.3: banco de 1,3 $\mathrm{m}$ constituido por calizas de tonalidad beige, oncolíticas y con bioclastos, que hacia techo cambian a facies más tobáceas y más claras. Se observa porosidad de disolución de tipo vug y rellenos de arcilla. La parte superior del banco se corresponde con calizas tobáceas, identificándose cementos esparíticos de calcita en huecos de disolución.

Tramo 2 - intermedio (5,8 metros de potencia):

2.1: banco de $2,5 \mathrm{~m}$ constituido por capas de caliza margosa homogénea que presenta una tonalidad clara. Se observan abundantes huellas de raíces verticales.

2.2: banco de 1,3 $\mathrm{m}$ de espesor formado por la alternancia de capas de calizas tobáceas y calizas oncolíticas, predominando estas últimas hacia techo del banco. Ambas facies muestran una tonalidad beige, rasgos de disolución y rellenos arcillosos.

2.3: banco de $2 \mathrm{~m}$ de potencia en el que se identifican dos capas de calizas oncolíticas, con potencia muy similar, separadas por una superficie erosiva. En la capa inferior, de tonalidad beige, se observan abundantes oncolitos y ciertos rasgos de disolución. En la capa superior, la facies es oncolítica, muy esparítica, de tonalidad grisácea y se identifican gravels, estructuras algares y bioclastos.

Tramo 3 - superior (8,2 $\mathrm{m}$ de potencia):

3.1: banco de $1 \mathrm{~m}$ de capas de calizas tobáceas con raíces y envueltas algares, muy cementadas y con tonalidad clara.

3.2: banco de 2,2 $\mathrm{m}$ de capas de calizas tobáceas con envueltas algares, porosas y de tonalidad clara. Se aprecian gravels dispersos y porosidad tipo vug rellena de material arcilloso.

3.3: banco de $5 \mathrm{~m}$ constituido por capas de calizas oncolíticas de tonalidad oscura muy cementadas. Hacia la base se puede observar una zona karstificada con relleno brechoide y arcillas. Se observan secciones de raíces y abundantes gravels.

A partir de los caracteres petrográficos observados en muestra de mano en la caliza tomada de las fachadas del Antiguo Hospital (figura 8), se establece 

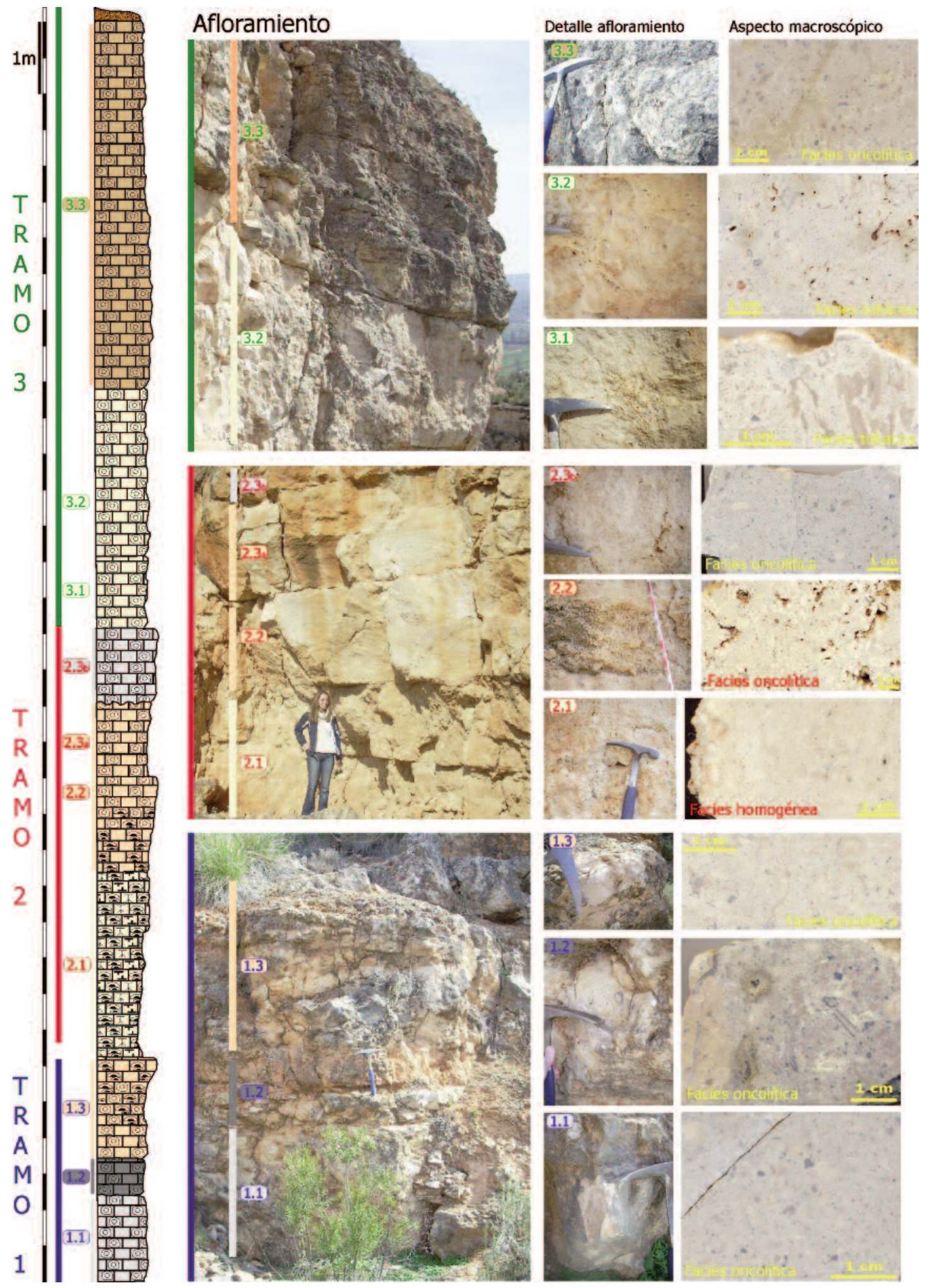

Figura 7. Columna estratigráfica levantada en el antiguo frente de explotación en las canteras de Cornicabra. Se muestran los tres tramos establecidos así como el aspecto in situ de la práctica totalidad de los bancos de caliza diferenciados y el aspecto macroscópico de las facies identificadas (mayoritariamente facies oncolíticas). Localización: $40^{\circ} 1352 \mathrm{~N} / 3^{\circ} 2747 \mathrm{O}$.

Figure 7. Stratigraphic column of the re-discovered quarry front of Cornicabra ancient quarries. The three beds identified are shown, with photographs of practically all the differentiated limestone layers and macroscopic images of the facies identified (primarily oncolitic). Location: $40^{\circ} 13^{\prime} 52^{\prime \prime} N / 3^{\circ} 27^{\prime} 47^{\prime \prime} W$ 
su correspondencia bien con calizas micríticas que presentan porosidad de disolución (tipo vug) -con cemento esparítico de calcita o tapizada con material arcilloso- (facies homogénea), bien con calizas constituidas por construcciones biogénicas, fundamentalmente estructuras estromatolíticas (facies estromatolítica-oncolítica). En los cuatro testigos extraídos, la caliza muestra una tonalidad beige o amarillenta y un elevado grado de cementación.

Atendiendo a los caracteres texturales y composicionales, existe cierta similitud entre la facies oncolítica de los bancos $1.1,1.2$ y $2.3 \mathrm{~b}$ establecidos en el antiguo frente de cantera (figura 7) con el testigo $C$ de fachada -facies estromatolítica-oncolítica- (figura 8c), así como entre la caliza del banco 2.1 (figura 7) y el testigo A (figura 8a) de fachada -facies homogénea-.

\section{Comparación petrográfica de la caliza del afloramien- to y de las fachadas}

Se realiza el análisis petrográfico atendiendo comparativamente a los caracteres texturales y composicio-
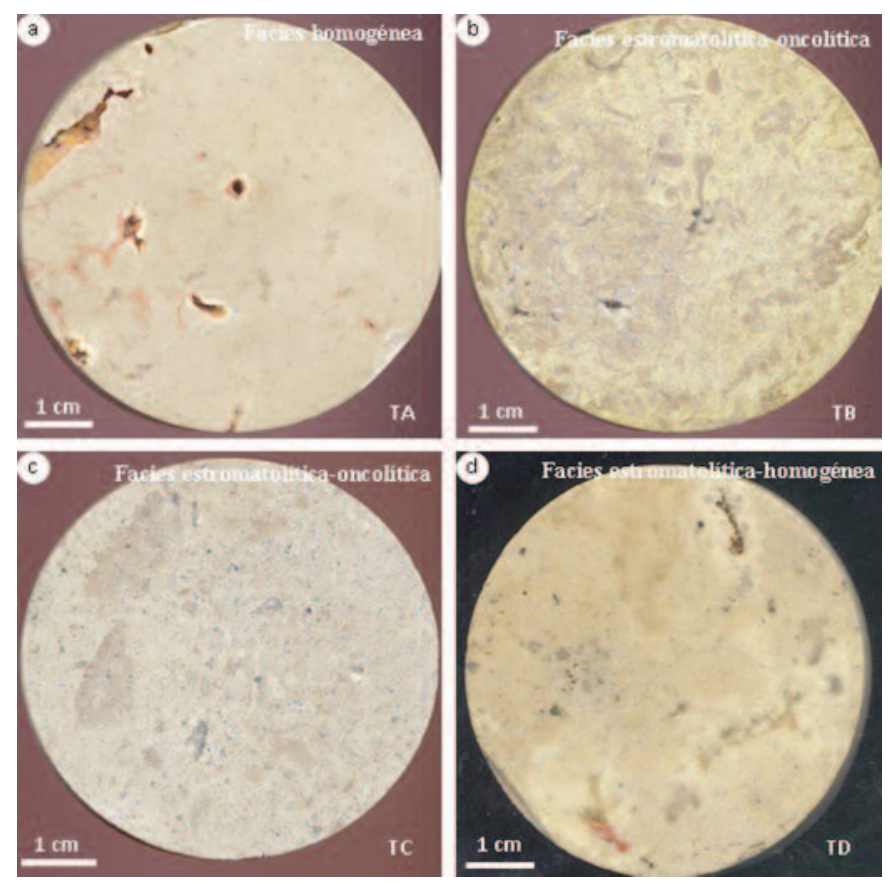

Figura 8. Aspecto macroscópico de la caliza empleada en la construcción de las fachadas del Antiguo Hospital de Maudes, estableciéndose su correspondencia con facies características de ambientes fluviolacustres.

Figure 8. Macroscopic images of the limestone used to build the facades of the former Maudes Workers' Hospital and its correspondence with facies characteristic of fluvial-lacustrine environments. nales de las muestra de caliza tomadas en fachada y en el afloramiento observados mediante Microscopía Óptica de Polarización (figura 9). Las partículas que constituyen el esqueleto son principalmente bioclastos $y$, en menor medida, intraclastos. Los bioclastos se corresponden con secciones longitudinales $y$ transversales de tallos de algas charáceas, con fragmentos o restos completos de moldes de ostrácodos y gasterópodos, y con estructuras de envueltas micríticas -propias de estructuras estromatolíticas y/o oncolíticas- desarrolladas principalmente sobre las secciones de charáceas (figuras 9a-9c).

El cemento se corresponde con cristales esparíticos de calcita que rellenan parcial o completamente la porosidad. Principalmente se identifica una porosidad intrapartícula, en el interior de los bioclastos y asociada a las secciones de charáceas, e interpartícula, cementando los constituyentes del esqueleto (figuras $9 \mathrm{a}$ y $9 \mathrm{~b}$ ). Se aprecian diversos crecimientos cristalinos de calcita, correspondientes a diferentes episodios o fases de cementación. Así, se observa el relleno micrítico de los poros (silt o limo vadoso), presentando los cristales una tonalidad amarillenta (figuras $9 \mathrm{c}$ y $9 \mathrm{~d}$ ), y cementos politexturales con distribución geopetal que cierran el poro. Se observan abundantes rellenos geopetales (figuras $9 a$ y $9 c$ ) y un material de coloración rojiza (figura $9 \mathrm{~d}$ ), que posiblemente se corresponda con geles de hierro. La matriz es micrítica, está constituida por peloides y se observan grietas cementadas por esparita, posiblemente relacionadas con la bioturbación del sedimento (figuras $9 \mathrm{e}$ y $9 \mathrm{f})$.

La facies oncolítica (cantera, figura 7), estromatolítica-oncolítica (testigos $\mathrm{B}$ y $\mathrm{C}$ de fachada, figuras $8 \mathrm{~b}$ y 8c) y estromatolítica-homogénea (testigo $D$ de fachada, figura 8d) se clasifican según Folk (1962) como una bioesparita de charáceas $y$ oncolitos $y$, según Dunham (1962), como un grainstone de charáceas y oncolitos. La facies homogénea (banco 2.1 del afloramiento en la figura 7 y testigo $A$ de fachada, figura 8a) se corresponde con una caliza micrítica con matriz peloidal predominante y escasos bioclastos (figura 9), clasificándose como una pelesparita (Folk, 1962) o grainstone de peloides (Dunham, 1962).

La descripción petrográfica de las muestras tomadas, en la cantera y en el edificio, permite comprobar la importante similitud que presentan (figura 9), resultando más significativa que para los caracteres texturales observados de visu en las muestras (figuras 7 y 8). Este estudio comparativo aporta un dato más para confirmar la procedencia de la caliza del Antiguo Hospital en las canteras de Cornicabra, tal y como señala la documentación consultada (LópezUrrutia, 1926). 

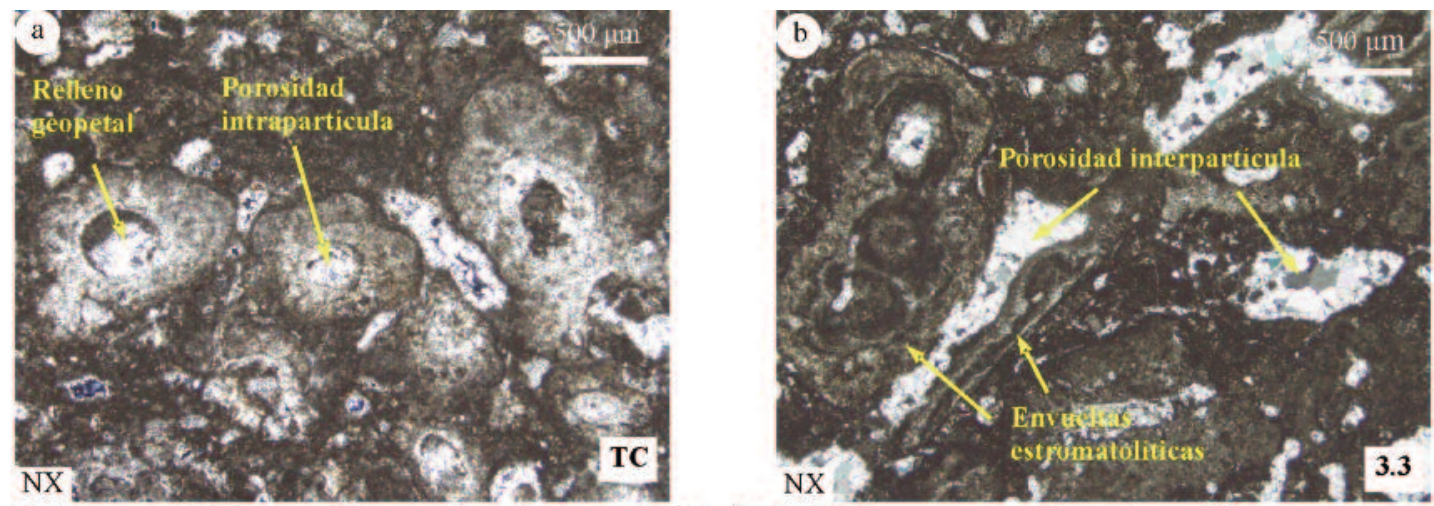

Facies estromatolítica-oncolítica: secciones transversales de tallosde charáceas con envueltas estromatolíticas.
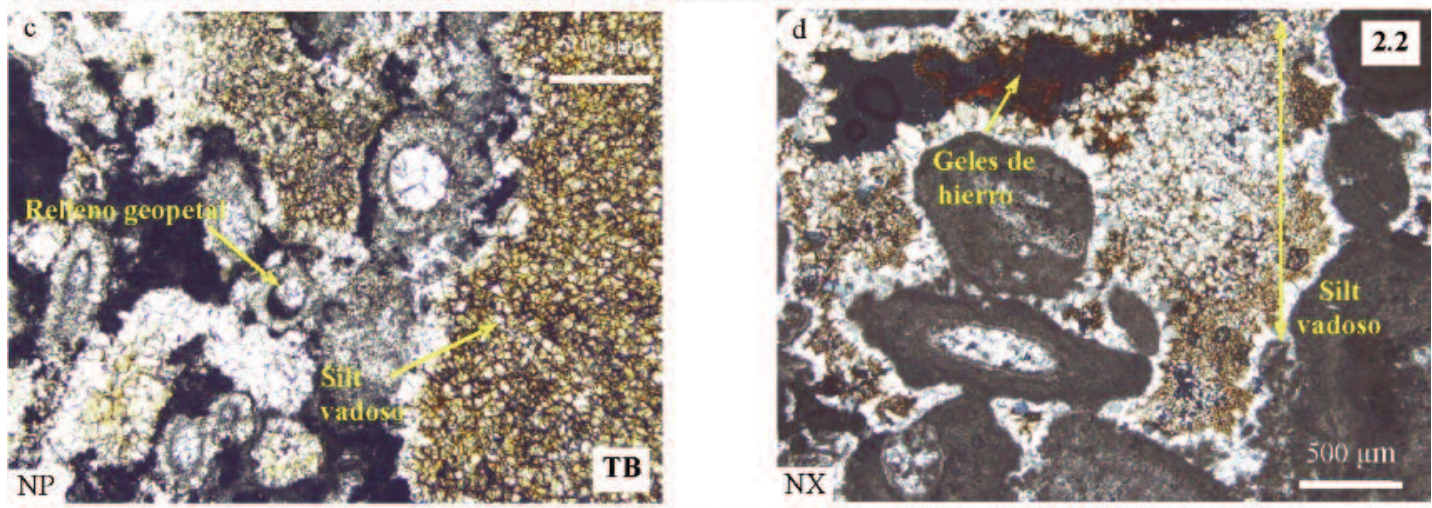

Facies estromatolitica-oncolitica: caliza biogénica de charáceas con diversos episodios de cementación.
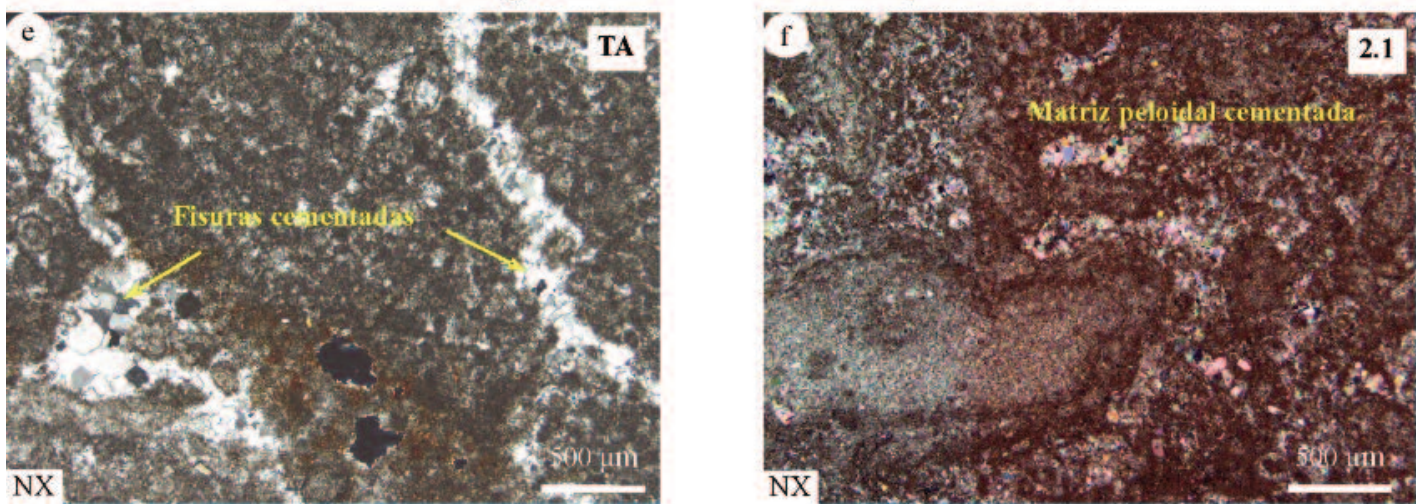

Facies homogénea: caliza micrítica con matriz peloidal.

Figura 9. Comparativa entre los principales caracteres petrográficos observados mediante Microscopía Optica de Polarización en la piedra caliza tomada de las fachadas del Antiguo Hospital (izda.) y de la cantera (dcha.). NP: nícoles paralelos, NX: nícoles cruzados.

Figure 9. Comparison of the major petrographic features observed by means of Optical Microscopy found in the limestone samples from the hospital (left) and the quarry (right). NP: plane nicols, NX: crossed nicols.

\section{Caracterización petrofísica de la caliza del Antiguo Hospital de Maudes}

En la tabla 3 se recogen los valores medios y de desviación estándar de los parámetros petrofísicos determinados en las probetas dimensionadas a partir de los testigos extraídos en la fachada del edificio (A, B, $C$ y D). En cuanto al color, las superficies de las pro- betas presentan valores de $L^{*}$ e IA similares y el valor de IB en el testigo A resulta negativo, de ahí los mayores valores en el IA, coordenada $b^{*}$ y saturación de color $\left(C^{*}\right)$. Los valores de velocidad de propagación de las ondas ultrasónicas resultan muy elevados ( $X V p>5800 \mathrm{~m} / \mathrm{s}$ ) y los índices de anisotropía presentan valores bajos ( $\Delta \mathrm{M} \%$ y $\Delta \mathrm{m} \%<2 \%$ ), siendo además muy similares entre sí. El valor medio del índice de 
rebote $(R)$ medido in situ en la superficie del sillar se corresponde con un valor de $56( \pm 1.6)$.

El valor de densidad real obtenido en los cuatro testigos ensayados $\left(2.68-2.69 \mathrm{~g} / \mathrm{cm}^{3}\right)$ es muy similar a la densidad de la calcita $\left(2.71 \mathrm{~g} / \mathrm{cm}^{3}\right)$, constituyente mineralógico principal de la piedra estudiada, y para la densidad aparente resultan valores ligeramente inferiores $\left(2.50-2.63 \mathrm{~g} / \mathrm{cm}^{3}\right)$. La diferencia en valor entre la densidad real y la densidad aparente no alcanza un valor superior a los $0.2 \mathrm{gr} / \mathrm{cm}^{3}$, señalando un bajo grado de porosidad en la caliza. Los índices de compacidad son muy elevados (XIC $>90 \%$ ), resultando este valor menor cuanto más bajo es el valor de densidad aparente, incrementándose la diferencia respecto a la densidad real $y$, por tanto, resultando mayor la porosidad accesible al agua.

Respecto a los parámetros hídricos, por un lado, la caliza muestra un bajo porcentaje de porosidad accesible al agua $(4.00 \pm 2.1 \%)$ y al mercurio $(2.5 \pm 1.0 \%)$ así como de cantidad máxima de agua que es capaz de absorber por saturación al vacío $(1.6 \pm 0.9 \%)$ y coeficiente de permeabilidad al vapor de agua $(0.06 \pm$ $0.07 \mathrm{~g} / \mathrm{m}^{2} \cdot 24 \mathrm{~h} \mathrm{mmHg}$ ). Por otro lado, en la caliza predomina la microporosidad frente a la macroporosi- dad accesible al mercurio y el porcentaje de agua absorbida por saturación es mayor cuanto mayor es la porosidad accesible al mercurio. El ángulo de contacto estático entre la superficie y el agua presenta un valor elevado, resultando un valor medio de $72,4^{\circ}{ }( \pm$ 0.5).

Las curvas de distribución porosimétrica muestran una tendencia unimodal (figura 10), presentando la mayoría de los poros tamaños medios de diámetro comprendidos entre 0.01 y $0.1 \mathrm{~m}$. Otra moda representativa se concentra en el intervalo 0.1-1 m, que resulta algo más significativa para el testigo $D$, cuya curva muestra cierta tendencia polimodal, revelando la presencia de poros con tamaños más diferentes.

\section{Discusión y resultados}

\section{Caliza de Morata de Tajuña versus caliza de Colmenar de Oreja a principios del siglo $X X$}

El abandono de las canteras de Colmenar de Oreja a finales del siglo XIX y primeros años del XX, más que al agotamiento de la caliza se debía a una cuestión

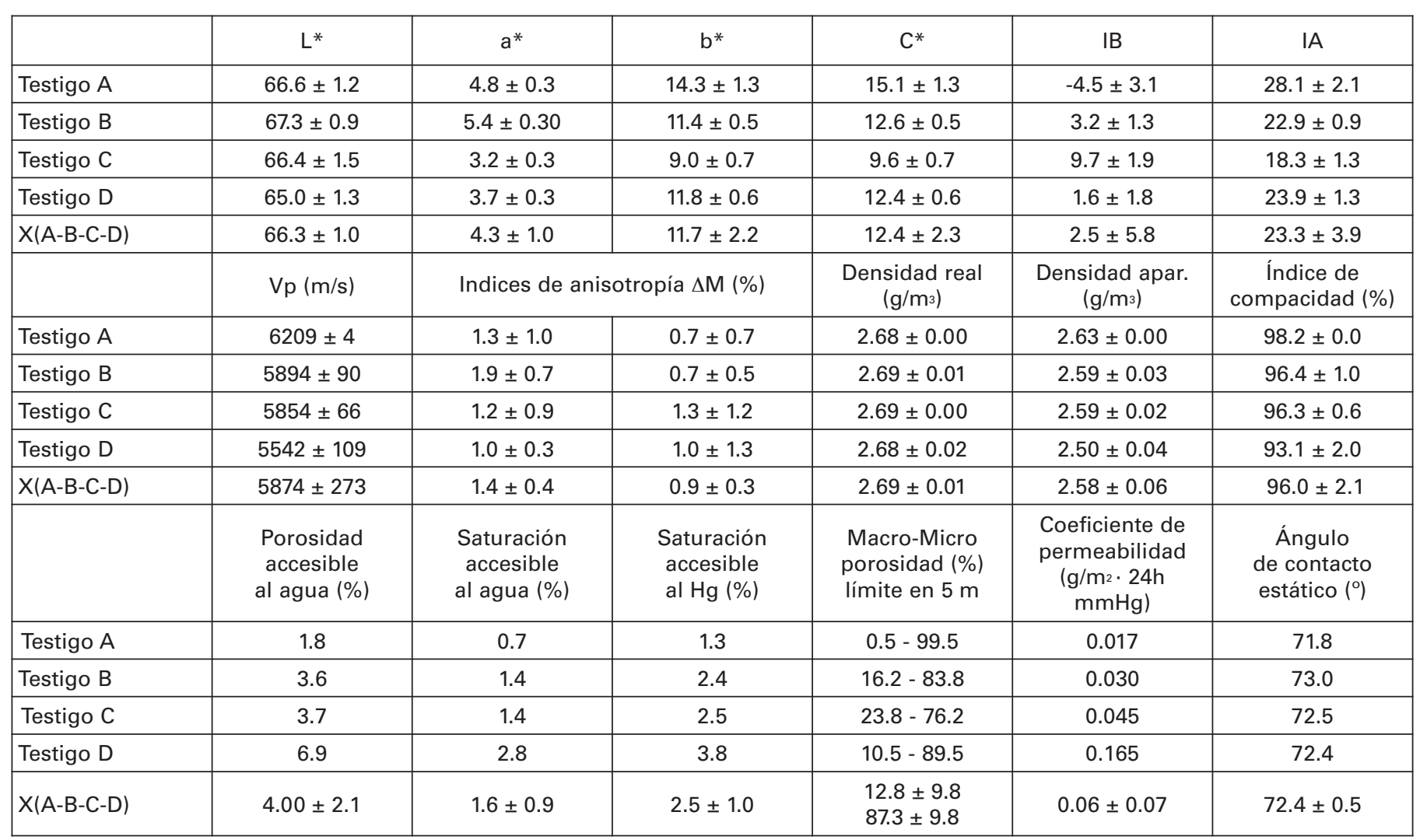

Tabla 3. Parámetros petrofísicos de la caliza del Antiguo Hospital, procedente de Morata de Tajuña, determinados en laboratorio.

Table 3. Laboratory-determined petrophysical properties of the Morata de Tajuña limestone from the former Maudes Workers' Hospital. 

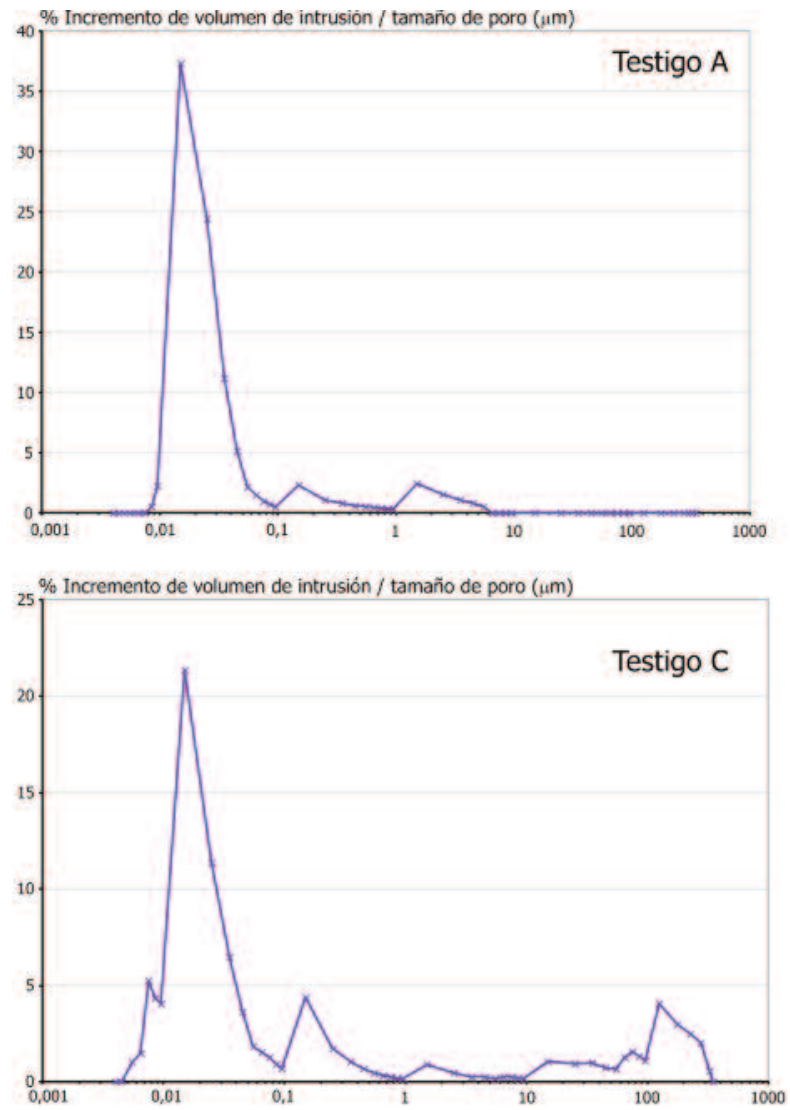
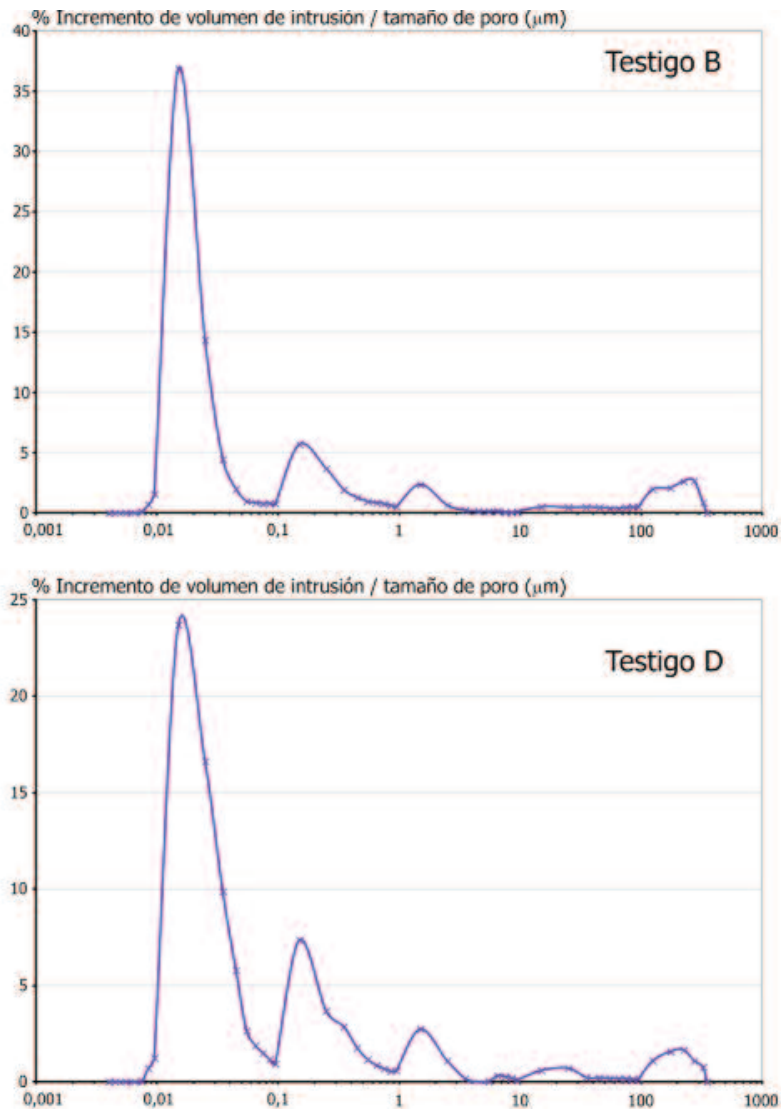

Figura 10. Curvas de distribución porosimétrica de la caliza tomada en las fachadas del Antiguo Hospital. Figure 10. Pore size distribution of the limestone sampled from the hospital facades.

económica. Así, el trazado ferroviario existente por entonces en el país posibilitaba que resultara mucho más rentable el empleo de calizas procedentes de zonas más lejanas. Ciertamente, las canteras de Colmenar de Oreja explotadas durante el XVIII estaban abandonadas $y$, aunque todavía quedaban reservas, no debía interesar la apertura de nuevos frentes. La llegada del ferrocarril a Colmenar de Oreja en 1903 reactivó las canteras, si bien se mantuvo el empleo de piedra foránea a la provincia pues seguía resultando más económica, de modo que, a principios del XX, la explotación de caliza en Colmenar de Oreja se mantuvo pero a un ritmo menor que antaño (Puche and Mazadiego, 1999).

A principios del siglo $X X$, ya era bien conocida la caliza de Morata de Tajuña, principalmente empleada para la fabricación de cal y cemento en el paraje del Valhondo. De hecho, como se ha indicado, cuando llega el tren a la localidad en 1901, se envió una comisión para el reconocimiento de las canteras (De la Torre and Paris, 1986). El documento realizado por la Sociedad Canteras de Cornicabra en 1907 refleja la importante actividad minera existente por entonces en las canteras del mismo nombre y el potencial que presentaba la caliza allí extraída, principalmente para el dimensionado de piezas de cantería. Además, en 1908 la Estadística Minera recoge una importante producción diaria de cal por la Sociedad de Cementos y Canteras de Valhondo (Puche and Mazadiego, 1999).

La explotación a principios del siglo XX en las canteras de Cornicabra de caliza destinada a piezas de cantería era muy favorable, resultando una piedra altamente competitiva. Por un lado, que apenas fuera necesario efectuar labores de desmonte permitía una extracción más sencilla que la caliza de Colmenar de Oreja. Por otro, el ferrocarril y el apeadero anexo a las canteras reducían los costes de transporte. De este modo, esta caliza resultaba económicamente ventajosa frente a otras calizas que se explotaban por entonces, tanto en la provincia como fuera de ella.

El apogeo de la caliza de las canteras de Cornicabra destinada al dimensionado de piedra para las construcciones madrileñas, puede situarse aproximadamente entre 1905 y 1925. Más tarde, la utilización de la misma se centró en su beneficio para la obtención de cal destinada a la industria del cemento, 
Pérez-Monserrat, E. M., et al., 2017. La caliza de Morata de Tajuña, Comunidad de Madrid:... Boletín Geológico y Minero, 128 (4): $963-988$

siendo muy puntual su empleo como elemento de cantería. La clara apuesta de Antonio Palacios por incorporar a sus construcciones materiales locales, como seña de identidad de los lugares donde construía, y el carácter tradicional del Antiguo Hospital, principalmente por estar construido en piedra y además con una piedra tradicional de la región, contribuyeron al impulso en la capital a principios del siglo $X X$ de un material que había sido muy poco utilizado $y$, por tanto, resultaba apenas conocido. Dada la considerable magnitud del inmueble, es muy posible que gran parte de las reservas de caliza de las canteras de Cornicabra tuviera como destino el Antiguo Hospital. De hecho, la construcción del inmueble (1909-1916) se incluye temporalmente en el periodo de tiempo durante el que la caliza de las canteras de Cornicabra fue principalmente explotada como piedra de cantería (1905-1925). Este aspecto participa de la excepcionalidad del Antiguo Hospital.

\section{Ambiente de formación y procesos diagenéticos}

Las facies caracterizadas en particular, y los carbonatos fluviolacustres del Mioceno Superior en general, se formaron en un ambiente lacustre somero, en donde se produce la rápida colmatación de la cuenca y los sedimentos carbonáticos sufren importantes modificaciones debido a fluctuaciones en el nivel de agua. Los procesos acontecidos en los estadios iniciales de la diagénesis, corresponden a la alternancia de periodos de encharcamiento con etapas de exposición subaérea.

El sedimento original debía presentar una tonalidad oscura, siendo la oxidación de la materia orgánica otra impronta de la desecación acontecida en la cuenca que, junto a los cementos diagenéticos, son los principales responsables del progresivo aclaramiento en la tonalidad del depósito original (García del Cura et al., 1996). También en condiciones de encharcamiento se produce la cementación de la matriz peloidal, rasgo característico de ambientes freáticos continentales, que implica una cementación temprana y que ha impedido el colapso de los peloides (Scholle and Ulmer-Scholle, 2003).

La existencia de cavidades con rellenos geopetales revela también un ambiente de sedimentación con alternancia de exposición subaérea y condiciones freáticas (Freytet and Plaziat, 1982). En ambientes vadosos tiene lugar la sedimentación interna o relleno micrítico de los poros (silt o limo vadoso), relacionándose el cemento de tonalidad amarillenta posiblemente con las condiciones vadosas así como con la existencia de arcillas y/o óxidos de hierro. El cemento freático suele terminar por sellar el poro vadoso, resultando cementos politexturales con distribución geopetal (García del Cura et al., 1994).

\section{Calidad de la caliza Morata de Tajuña y su comparati- va con la Caliza de Colmenar de Oreja}

Los caracteres petrológicos que presenta la caliza de Morata de Tajuña (de cantera y/o edificio), dependen del ambiente de formación y de los procesos diagenéticos acontecidos en la cuenca, configurando un material pétreo muy apropiado para ser utilizado como elemento portante, con elevada dureza superficial y resistencia. Además de resultar una piedra especialmente idónea para soportar esfuerzos a compresión, su comportamiento hídrico le otorga una elevada resistencia a la acción de los agentes de deterioro, especialmente al agua (en sus tres estados), por lo que presentará igualmente una elevada durabilidad.

Entre los rasgos texturales primarios, señalar la presencia de abundantes moldes de bioclastos (algas, gasterópodos y estructuras estromatolíticas y oncolíticas), que suponen una porosidad susceptible de cementación, y la ausencia de orientaciones preferentes. En cuanto a los procesos diagenéticos, principalmente destacar los procesos de cementación, que disminuyen la porosidad primaria, especialmente la porosidad intercristalina asociada a la micrita. En los cementos diagenéticos, señalar por un lado los relacionados con la diagénesis de enterramiento, caracterizados por la presencia de microcristales y por la cementación de la matriz peloidal. Por otro, los macrocristales de cemento freático, incluyendo los cementos politexturales con distribución geopetal (García del Cura et al., 1996). El valor medio de luminosidad $\left(X^{*}>66\right)$ responde al color claro de la calcita, constituyente mineralógico principal de la piedra caracterizada, así como a la oxidación de la materia orgánica. Además, los cementos freáticos son normalmente claros y su mineralogía se corresponde con calcita de bajo contenido en hierro (García del Cura et al., 1996), otorgando igualmente una tonalidad clara a las facies. Valores más elevados de croma $\left(C^{*}\right)$ e índice de amarillo (IA) medidos responden principalmente a la cementación vadosa, en la que ha tenido lugar el relleno micrítico de los poros con silt o limo vadoso, de coloración amarillenta.

La caliza ofrece muy buen comportamiento mecánico, reflejado principalmente por los elevados valores tanto de velocidad de propagación de los ultrasonidos ( $X V p>5500 \mathrm{~m} / \mathrm{s}$ ) como de dureza superficial a partir del índice de rebote obtenido $(X R=56)$, seña- 
lando en ambos casos igualmente una elevada compacidad del material (XIC > 90\%). El índice de rebote obtenido refleja una importante dureza superficial de la piedra (Aydin and Basu, 2005). Respecto a los índices de anisotropía, además de presentar bajos valores medios ( $\mathrm{X} \Delta \mathrm{M}$ y $\mathrm{X} \Delta \mathrm{m}<2 \%$ ), que resulten tan similares entre sí indica la inexistencia de una dirección preferente (Zezza, 1990), por lo que su puesta en obra no requeriría, en principio, colocar las piezas según una orientación determinada (Fort et al., 2011). Los similares valores entre densidad real y aparente indican una baja porosidad accesible al agua en la caliza ensayada (UNE-EN 1936:2006). Los elevados valores de $\mathrm{Vp}$ e índice de compacidad así como la similitud entre la densidad real y aparente se corresponden con bajos valores medios de porosidad accesible al agua $(4 \%)$, de agua de saturación $(1.6 \%)$ y de permeabilidad al vapor de agua $\left(<0.1 \mathrm{~g} / \mathrm{m}^{2} \cdot 24 \mathrm{~h} \mathrm{mmHg}\right)$.

La resistencia que presenta la caliza a la acción del agua queda establecida, por un lado, por la tendencia unimodal de las curvas de distribución porosimétrica, de modo que a menor variabilidad en los tamaños de poros, menos circulará por su interior. Por otro, por la concentración de la mayoría de los poros en el intervalo de diámetro de poros 0.01-0.1 m, tamaño que dificulta la circulación del agua en su interior. Ciertamente, a mayor numero de poros de pequeño tamaño, mayores son los efectos disruptivos causados por la cristalización de sales y/o acción del hielo- deshielo (Benavente et al., 2007; López-Arce et al., 2011; Fort et al., 2013; Freire-Lista et al., 2015). Ahora bien, estos efectos están principalmente relacionados con la presencia de poros capilares, cuyos tamaños se encuentran en el intervalo 1-100 m, y que resultan muy poco significativos en la caliza estudiada. El bajo coeficiente de permeabilidad $\left(0.06 \mathrm{~g} / \mathrm{m}^{2} \cdot 24 \mathrm{~h} \mathrm{mmHg}\right)$ indica la dificultad que presenta la piedra a la entrada en su interior del vapor de agua. No obstante, al resultar tan impermeable al vapor de agua, dificulta también la salida de humedad procedente, por ejemplo, del interior de los muros. La caliza presenta una importante capacidad hidrofugante de forma natural, pues el valor medio de ángulo de contacto es superior a $70^{\circ}$.

Comparando los parámetros petrofísicos de la caliza empleada en la construcción del Antiguo Hospital, procedente de Morata de Tajuña, con los valores de la caliza de Colmenar de Oreja (Fort et al., 1996a, 2011, 2013 y 2015), se observa que en general ambas presentan un comportamiento petrofísico parecido (tabla 4). De este modo, tienen valores muy similares en la velocidad de propagación a los ultrasonidos, densidad (real y aparente), porosidad accesible (al agua y al mercurio), porcentaje de agua absorbida por saturación al vacío, macro y microporosidad. Las dos calizas muestran bajos valores de anisotropía total y de coeficiente de permeabilidad al vapor de agua, si bien ambos valores resultan infe-

\begin{tabular}{|c|c|c|}
\hline & Caliza Colmenar Oreja* & Caliza Morata Tajuña \\
\hline Indice de blancura (IB) & 22.6 & 2.5 \\
\hline $\mathrm{Vp}(\mathrm{m} / \mathrm{s})$ & 5800 & 5874 \\
\hline Anisotropía total (dM\%) & 2.3 & 1.4 \\
\hline Densidad real $\left(\mathrm{g} / \mathrm{cm}_{3}\right)$ & 2.7 & 2.69 \\
\hline Densidad aparente $\left(\mathrm{g} / \mathrm{cm}^{3}\right)$ & 2.6 & 2.58 \\
\hline Porosidad acces. agua (\%) & 3.2 & 4.0 \\
\hline Saturación (\%) & 1.3 & 1.6 \\
\hline Porosidad acces. $\mathrm{Hg}(\%)$ & 3.6 & 2.52 \\
\hline Ángulo contacto estático $\left({ }^{\circ}\right)$ & 60.8 & 72.4 \\
\hline
\end{tabular}

*Tomado de Fort et al., 1996a, 2011, 2013, 2015.

Tabla 4. Comparativa de algunos parámetros petrofísicos de las calizas de Colmenar de Oreja y de Morata de Tajuña. Table 4. Colmenar de Oreja and Morata de Tajuña limestones: comparison of petrophysical properties. 
Pérez-Monserrat, E. M., et al., 2017. La caliza de Morata de Tajuña, Comunidad de Madrid:... Boletín Geológico y Minero, 128 (4): $963-988$

riores para la caliza de Morata de Tajuña. Ahora bien, resultan sensiblemente diferentes en cuanto a su cromatismo se refiere, mostrando la caliza de Colmenar de Oreja una luminosidad e índice de blancura considerablemente mayores y la caliza de Morata deTajuña un índice de amarillo más elevado. También, la caliza de Morata de Tajuña tiene una capacidad hidrofugante natural mayor que la caliza de Colmenar de Oreja, al presentar un ángulo de contacto estático más elevado.

Por tanto, al compartir origen geológico y procesos diagenéticos, ambas calizas resultan materiales de muy elevada calidad y muy adecuados para configurar elementos constructivos portantes resistentes a la acción de los agentes de deterioro. Aunque la caliza de Morata de Tajuña no presenta la blancura de la caliza de Colmenar de Oreja, debido principalmente al relleno micrítico de los poros en episodios de cementación vadosa por limo de coloración amariIlenta, los valores obtenidos en algunos de los parámetros señalan que presenta un comportamiento mecánico e hídrico incluso mejor que la caliza de Colmenar de Oreja, cuya calidad es ya de por sí muy elevada (Fort et al., 2011, 2013 y 2015).

Respecto a los caracteres petrográficos, la principal diferencia observada entre la caliza de Colmenar de Oreja y la de Morata de Tajuña es la abundancia muy significativa en la primera de tallos y oogonios de charáceas así como de gasterópodos (Fort et al., 1996a, 2011, 2013 y 2015), si bien en la caliza de Morata deTajuña se han reconocido igualmente estos bioclastos, siendo especialmente abundantes en algunas de las secciones delgadas observadas.

\section{Conclusiones}

Tanto la formación de las rocas y posteriores procesos geológicos acontecidos -que determinan sus propiedades petrológicas $\mathrm{y}$, por tanto, su comportamiento y calidad para desempeñar una determinada función constructiva-, como los diferentes aspectos históricos en los que se encuadra su extracción y transporte -que principalmente determinan los costes- suponen factores decisivos a la hora de seleccionar el uso de una u otra piedra tradicional en una época determinada.

El uso en la ciudad de Madrid de la caliza de las canteras de Cornicabra (Morata de Tajuña) a principios del siglo $X X$ se debió principalmente a la relativa facilidad que presentaba su extracción y al apeadero que se construyó a pie de cantera para el traslado directo de la piedra a la capital mediante el ferrocarril delTajuña, aspectos que abarataban consi- derablemente los costes. Además de resultar entonces una piedra económicamente muy competitiva, frente a la caliza de Colmenar de Oreja o piedras procedentes de otras provincias que se estaban empleando entonces en la ciudad, presenta una calidad tan elevada como la caliza de Colmenar de Oreja, si bien carecía de su prestigio, belleza y blancura.

La elevada calidad de la caliza objeto de estudio queda establecida a partir de sus propiedades petrofísicas. Principalmente, elevados valores de dureza superficial $(X R=56)$, de velocidad de propagación a los ultrasonidos (XVp > $5500 \mathrm{~m} / \mathrm{s}$ ) y de compacidad (XIC $>90 \%$ ) y bajos porcentajes de porosidad accesible al agua $(4 \%)$ y de agua absorbida por saturación al vacío $(1.6 \%)$ como permeabilidad al vapor de agua $\left(<0.1 \mathrm{~g} / \mathrm{m}^{2} \cdot 24 \mathrm{~h} \mathrm{mmHg}\right)$. Destacar además su baja anisotropía ( $X \Delta \mathrm{M}$ y $\mathrm{X} \Delta \mathrm{m}<2 \%$ ), importante capacidad hidrofugante que de forma natural presenta la caliza (ángulo de contacto $>70^{\circ}$ ) y el intervalo porosimétrico $(0.01-0.1 \mathrm{~m})$ en el que se concentra el porcentaje de microporosidad (87.3\%).

El empleo a principios del siglo XX de la caliza de Morata de Tajuña como piedra tradicional en Madrid, en una época en la que la piedra de la región estaba siendo sustituida por materiales de construcción foráneos, y que se trate de una caliza ciertamente desconocida en la ciudad, en gran parte por el corto periodo de tiempo durante el que fue utilizada como piedra de cantería, otorgan sin duda un valor añadido a la caliza de las canteras de Cornicabra. El Antiguo Hospital de Maudes, obra de Antonio Palacios, supone un ejemplo excepcional de patrimonio construido en Madrid con caliza explotada en las canteras de Cornicabra. La construcción del inmueble está muy vinculada con el empleo de esta caliza como piedra de cantería en la ciudad a principios del siglo $X X$, respondiendo este uso tal vez en parte a la construcción del inmueble.

\section{Agradecimientos}

Los autores agradecen a José María la Calle, antiguo subdirector de la Dirección General de Arquitectura y Urbanismo de la Comunidad de Madrid, y a José María Cabrera, gerente de la empresa CPA, S.A., la ayuda y facilidades prestadas para el acceso al Antiguo Hospital y toma de muestras. Se agradece la imagen del Antiguo Hospital prestada por el Centro Documentación Especializada de la Consejería de Medio Ambiente y Ordenación del Territorio del Comunidad de Madrid y el excepcional documento cedido por el Archivo Histórico Ferroviario del Museo del Ferrocarril de Madrid - Fundación de los 
Ferrocarriles Españoles, dando las gracias especialmente a Raquel Letón. Se dan las gracias a Marián Barajas, del Departamento Petrología y Geoquímica de la Facultad de Ciencias Geológicas (UCM), por la confección de las láminas delgadas, así como a César Chenel y Javier Rodríguez, antiguos técnicos del Laboratorio de Petrofísica del Instituto de Geociencias IGEO (CSIC, UCM) que realizaron los ensayos normalizados. Se agradece la financiación de los Programas Geomateriales (P2009/MAT_1629) y Geomateriales 2 (S2013/MIT_2914), financiados por la Comunidad de Madrid y el Fondo Social Europeo, y del grupo de investigación de la UCM Petrología Aplicada a la Conservación del Patrimonio (ref. $n^{\circ} 921349$ ). Finalmente, se agradecen los comentarios de los revisores anónimos, cuyas sugerencias han supuesto la mejora del trabajo presentado.

\section{Referencias}

Alonso-Zarza, A.M., Wright, V.P., Calvo, J.P. and García del Cura, M.A. 1992. Soli-landscape relationships in the middle Miocene of the Madrid Basin. Sedimentology, 39, 1735.

Álvarez de Buergo, M. and González-Limón, T. 1994. Estudio del método de la medida de la velocidad de propagación del sonido y su aplicación a edificios históricos. Ingeniería Civil, 94, 69-74.

Apartadero de Cornicabra. Proyecto de casilla para el guarda del cargadero de piedra, km 39,423. Compañía del Ferrocarril del Tajuña. 1905. Sig. Caja 26/21860. Archivo General de la Administración, Alcalá de Henares, Comunidad de Madrid.

ASTM E313:2010. Standard practice for calculating yellowness and whiteness indices from instrumentally measured color coordinates. American Society forTesting and Materials.

ASTM D5873:2000. Standard test method for determination of rock hardness by rebound hammer method. American Society forTesting and Materials.

Aydin, A. and Basu, A. 2005. The Schmidt hammer in rock material characterization. Engineering Geology, 81, 1-14.

Benavente, D., Cueto, N., Martinez-Martinez, J., Garcia del Cura, M.A. and Cañaveras, J.C. 2007. The influence of petrophysical properties on the salt weathering of porous building rocks. Environmental Geology, 39, 105113.

Boletín de la Revista de Obras Públicas. 1896. Canteras españolas. Caliza de Colmenar, 145-147.

Bustillo, A. 1980. Petrografía y medios sedimentarios de la caliza del páramo (prov. de Madrid). Boletín Geológico y Minero, XCl-III, 503-514.

Calvo, J.P., Ordóñez, A., García del Cura, M.A., Hoyos, M., Alonso-Zarza, A.M., Sanz, E. and Rodríguez Aranda, J.P. 1989. Sedimentología de los complejos lacustres miocenos de la Cuenca de Madrid. Acta Geológica Hispana, 24, 281-298.
Castells, J. and De la Concha. S. 1951. Memoria Hoja n ${ }^{\circ} 583$ (Arganda). Mapa Geológico de España. Escala 1:50.000. Instituto Geológico y Minero de España, Madrid.

Commission Internationale de I'Eclairage, CIE (1978): CIE Recommendations on uniform color spaces, color-difference equations and psychometric color terms. Colorimetry, supplement, 2-15.

Dapena, J.E., Ordóñez, S. and García del Cura, M.A. 1989. Estudio de las rocas calizas utilizadas durante los siglos XVIII y XIX en la construcción de Palacios de Madrid. Ingeniería Civil, 71, 67-77.

De Diego Arribas, J. 1891. Morata de Tajuña, tomo XII. Biblioteca de la Revista llustrada la Provincia, 96 pp.

De Pablos, F. 1891. Colmenar de Oreja, Madrid. Biblioteca de la Provincia de Madrid, Diputación provincial, Madrid, $54 \mathrm{pp}$.

De Prado, C. 1864. Descripción Física y Geológica de la Provincia de Madrid. Junta General de Estadística, Madrid, 220 pp.

De la Torre, J.A. and Paris, A. 1986. Centenario del Tren de Arganda: 1886-1986. Ayuntamiento de Arganda del Rey, $71 \mathrm{pp}$.

De la Torre, J.A. 1999. Historia de la Villa de Morata de Tajuña. Ayuntamiento de Morata de Tajuña, 507 pp.

Dunham, F.J. 1962. Classification of carbonate rocks according to depositional texture. In: Ham, W.E. (ed.), Classification of carbonate rocks. American Association of Petroleum Geologists Memoirs, 1, 108-121.

Echevarría, M. and García, J.I. 1996. El sector de la piedra natural. In: López Jimeno, C. (ed.), Manual de rocas ornamentales. Prospección, explotación, elaboración y colocación. Laboratorio Oficial para Ensayo de Materiales de Construcción, Getafe, Madrid, 25-40.

Esbert, R.M., Alonso F.J. and Ordaz, J. 2008. La petrofísica en la interpretación del deterioro y la conservación de la piedra de edificación. Trabajos de Geología, Universidad de Oviedo, 28, 87-95.

Estadística Minera 1909-1930. Consejo de la Minería y Ministerio de Fomento, Madrid.

Fernández-Navarro, L. 1907. Excursión desde el valle del Tajuña al del Tajo. Boletín de la Real Sociedad Española de Historia Natural, tomo VII, 136-139.

Fernández-Navarro, L. and Carandell, J. 1914. El borde de la meseta terciaria de Alcalá de Henares. Boletín de la Real Sociedad Española de Historia Natural, Tomo XIV, 329334.

Folk, R.L. 1962. Spectral subdivision of limestone types. In Ham, W.E. (ed.), Classification of Carbonate Rocks-A Symposium. American Association of Petroleum Geologists Memoir, 1, 62-84.

Fort, R., Mingarro, F. and López de Azcona, M.C. 1996a. Petrología de los materiales de construcción del Palacio Real de Madrid. Geogaceta, 20, 1236-1239.

Fort, R. 1996b. Análisis del sistema poroso de las rocas. In: Mingarro, F. (ed.), Degradación y Conservación del Patrimonio Arquitectónico. Editorial Complutense, 227236.

Fort, R. 1996c. El agua en los materiales pétreos: comportamiento hídrico. In: Mingarro, F. (ed.), Degradación y 
Pérez-Monserrat, E. M., et al., 2017. La caliza de Morata de Tajuña, Comunidad de Madrid:... Boletín Geológico y Minero, 128 (4): $963-988$

Conservación del Patrimonio Arquitectónico. Editorial Complutense, 237-248.

Fort, R., Bernabeú, A., García del Cura, M.A., López de Azcona, M.C., Ordóñez, S. and Mingarro, F. 2002. La Piedra Novelda: una roca muy utilizada en el patrimonio arquitectónico. Materiales de Construcción, 52 (266), 1932.

Fort, R., Varas, M.J., Álvarez de Buergo, M. and Freire, D.M. 2011. Determination of anisotropy to enhance the durability of natural stone. Journal of Geophysics and Engineering, 8, 132-144.

Fort, R., Álvarez de Buergo, M., Pérez-Monserrat, E.M., Gomez-Heras, M., Varas, M.J. and Freire, D.M. 2013. Evolution in the use of natural building stone in Madrid, Spain. Quarterly Journal of Engineering Geology and Hydrogeology, 46, 421-429.

Fort, R., Varas-Muriel, M.J., Alvarez de Buergo, M. and Perez-Monserrat, E.M. 2015. Colmenar Limestone, Madrid, Spain: considerations for its nomination as a Global Heritage Stone Resource due to its long term durability. In: Pereira, D., Marker, B.R., Kramar, S., Cooper, B.J. and Schouenborg, B.E. (eds.), Global Heritage Stone: Towards International Recognition of Building and Ornamental Stones. Geological Society, Special Publications, London, 407 (1), 121-135.

Freire-Lista, D., Fort, R. and Varas-Muriel, M.J. 2015. Freezethaw fracturing in building granites. Cold Regions Science and Technology, 113, 40-51.

Freytet, P. and Plaziat, J.C. 1982. Continental carbonate sedimentation and pedogenesis: Late Cretaceous and Early Tertiary of southern France. In: Purser, B.H. (ed.), Contributions to Sedimentology, 12. Schweizerbartsche Verlagsbuchhandlung, Stuttgart, $213 \mathrm{pp}$.

Friedman, G.M. 1959. Identification of carbonate minerals by staining methods. Journal of Sedimentary Petrology, 29, 87-97.

García del Cura, M.A., Ordóñez, S. and González, J.A. 1991. Los carbonatos biogénicos de los episodios terminales del relleno neógeno de la cuenca de Madrid. I Congreso del Grupo Español del Terciario, Vic, 136-139.

García del Cura, M.A., Ordóñez, S., Dapena, E. and González, J.A. 1994. Las canteras de calizas en los interfluvios de los ríos Jarama-Tajuña-Tajo en la Comunidad de Madrid: valoración de recursos. Boletín Geológico y Minero, 105 (6), 575-590.

García del Cura, M.A., Ordóñez, S., Fort, R. and Pina, J.A. 1996. El "Crema Marfil" (Pinoso, Alicante) II. Criterios petrográficos de calidad. Geogaceta, 20 (3), 731-734.

García-Fernández, J. 1948. Colmenar de Oreja: la industria de tinajas y la explotación de canteras. Estudios Geográficos, 9 (33), 649-665.

García de Miguel, J.M., Sánchez Castillo, L., Puche, O. and González-Aguado, M.T. 1992. Study of the Monumental Stone from Madrid District. 7th International Congress on Deterioration and Conservation of Stone, Lisboa, 4756.

Gomez-Heras, M. and Fort, R. 2004. Localización de canteras de materiales no tradicionales en la arquitectura de
Madrid: la Cripta de la Catedral de Santa María la Real de la Almudena. Materiales de Construcción, 54, 33-49.

González-Amezqueta, A. 1967. La arquitectura de Antonio Palacios. Arquitectura, 106, 1-74.

González-Yanci, M.P. 1974. Los accesos ferroviarios a Madrid. Su impacto en la geografía urbana de la ciudad. Instituto de Estudios Madrileños, Madrid, 520 pp.

Guydader, J. and Denis, A. 1986. Propagation des ondes dans les roches anisotropes sous contrainte évaluation de la qualité des schistes ardoisiers. Bulletin of Engineering Geology and the Environment, 33, 49-55.

Hernández-Pacheco, F. 1923. Geología de la Cuenca del Tajuña. Asociación Española para el Progreso de las Ciencias, tomo VI, 137-143.

Hernández-Pacheco, E. and Hernández-Pacheco, F. 1926. Aranjuez y el territorio sur de Madrid. XIV Congreso Geológico Internacional, Madrid. Excursión B-3.

Hernández-Pacheco, F. 1945. Materiales litológicos del territorio de Madrid empleados en la construcción. Las Ciencias, X (4), 959-982.

Hurtado, C. 1991. Colmenar de Oreja y su entorno. Graneas Zenit, Madrid, 633 pp.

Jiménez de Gregorio, F. 1978. Apunte geográfico-económico de los pueblos de la provincia de Madrid en el año 1752. Anales del Institutos de Estudios Madrileños, tomo XXII, 259-300.

Jiménez-Vega, M. and Polo, F. 2002. Los fracasos del ferrocarril madrileño: los casos del ferrocarril MadridAlmorox y del ferrocarril del Tajuña. II Congreso de Historia Ferroviaria, Siglo y Medio de Ferrocarriles, Aranjuez, 105-128.

Kindelan, J.A. and Cantos, J. 1946. Memoria Hoja n606 (Chinchón). Mapa Geológico de España. Escala 1:50.000. Instituto Geológico y Minero de España, Madrid.

López-Arce, P., Fort, R, Gómez-Heras, M, Pérez-Monserrat, E.M. and Varas-Muriel, M.J. 2011. Preservation strategies for avoidance of salt crystallisation in El Paular Monastery cloister, Madrid, Spain. Environmental Earth Sciences 63 (7-8), 1487-1509.

Madoz, P. 1848. Diccionario Geográfico Estadístico Histórico de España y sus posesiones en ultramar, tomo $\mathrm{XI}$, Madrid.

Martín, S. 1994. Materiales pétreos tradicionales de construcción en Madrid. Tesis doctoral, Universidad Politécnica de Madrid, 833 pp.

Martínez, J. 2000. La industria de la piedra natural en la Comunidad de Madrid. Roc Maquina, 61, 74-79.

Megías, A.G., Ordóñez, S. and Calvo, J.P. 1983. Nuevas aportaciones al conocimiento geológico de la Cuenca de Madrid. Revista de Materiales y Procesos Geológicos, 1, 163-191.

Menduiña, J. and Fort, R. (coord.) 2005. Las piedras utilizadas en la construcción de los Bienes de Interés Cultural de la Comunidad de Madrid anteriores al siglo XVIII. Instituto Geológico y Minero de España, Madrid, 132 pp.

Muñoz de Torres, A. 1993. Morata de Tajuña: lo que sé de mi pueblo. Ayuntamiento de Morata de Tajuña, 198 pp. 
Pérez-Monserrat, E. M., et al., 2017. La caliza de Morata de Tajuña, Comunidad de Madrid:... Boletín Geológico y Minero, 128 (4): $963-988$

NORMAL 21/85, 1985. Permeabilità al vapor d'acqua. NORmalizzazione MAteriali Lapidei, Centro Nazionale della Ricerca e Istituto Central del Restauro.

Ordóñez, S. and García del Cura. M.A. 1983. Recent and tertiary fluvial carbonates in Central Spain. In: Collinson, J.D and Lewin, J.D. (eds.), Modern and Ancient Fluvial Sistems, Special Publication of International Association of Sedimentologists, Gent, 6, 485-497.

Ordóñez, S. and García del Cura. M.A. 1994. Deposition and diagenesis of sodium-calcium sulphate salts in the Tertiery saline lakes of the Madrid Basin, Spain. In: Renaut, R.W. and Last, W.M. (eds.), Sedimentology and Geochemistry of Modern and Ancient Saline Lakes, Special Publication of Society of Economic Paleontologists and Mineralogists, Tulsa, 50, 220-238.

Ordóñez, S., García del Cura, M.A., Fort, R. and Pina, J.A. 1996. El "Crema Marfil" (Pinoso, Alicante) I. Características petrofísicas y parámetros de durabilidad. Geogaceta, 20 (3), 727-730.

Pereira, D. and Baltuille, J.M. 2014. Documenting natural stone to preserve our cultural and architectonic heritage. Materiales de Construcción, 64 (314), ed002.

Perez-Monserrat, E.M., Alvarez de Buergo, M., GomezHeras, M., Varas-Muriel, M.J. and Fort, R. 2013. An urban geomonumental route focusing on the petrological and decay features of traditional building stones used in Madrid, Spain. Environmental Earth Sciences, 69, 10711084.

Pérez-Monserrat, E.M. (2015). Caracterización, deterioro y limpieza de los materiales pétreos del Antiguo Hospital de Jornaleros de Madrid. Tesis doctoral, Universidad Complutense de Madrid, 524 pp.

Pérez-Monserrat, E.M., Fort, R., Varas-Muriel, M.J. and Álvarez de Buergo, M. 2017. Heritage value of building materials: Former Workers Hospital of Maudes, Madrid (Spain) case study. Ge-Conservacion, 11, 17-24.

Puche, O. and Mazadiego, L.F. 1999. Explotaciones históricas de calizas en la Comunidad de Madrid: Colmenar de Oreja. IV Sesión Científica de la Sociedad Española para la Defensa del Patrimonio Geológico y Minero, Bélmez, 91-104.

Quirantes, J. 1968. Estudio sedimentológico de las calizas del Terciario Continental. Boletín del Instituto de Estudios Asturianos, Suplemento de Ciencias, 14, 107111.

Riba, O., Arévalo, P. and Leiva, A. 1969. Estudio sedimentológico de las calizas del Páramo del terciario de la cubeta del Tajo. Boletín Geológico y Minero, 80, 525-537.

RILEM 1980. Recommended tests to measure the deterioration of stone and to assess the effectiveness of treatment methods. Commission 25 PEM (Protection et erosion des monuments). The International Union of Laboratories and Experts in Construction Materials, Systems and Structures. Materials and Structures, 75, 175-253.

Rodríguez, A.L. 2003. El ferrocarril del Tajuña, de gran proyecto ferroviario a línea de metro. Via Libre, la Revista del Ferrocarril, 05/06/2017, http://www.vialibreffe.com/noticias.asp?not $=1140$

Rodríguez-Aranda, J.P., Calvo, J.P. and Ordóñez, S. 1991. Transición de abanicos aluviales a evaporitas en el Mioceno del borde oriental de la Cuenca de Madrid (Sector de Barajas de Melo-Illana). Revista de la Sociedad Geológica de España, 4, 33-50.

Rodríguez-Navarro, C. and Sebastián-Pardo, E. 1994. Técnicas de análisis del sistema poroso de materiales pétreos ornamentales: usos y limitaciones. Ingeniería Civil, 96, 130-142.

Royo, J. 1929. Datos para el estudio de la geología de la provincia de Madrid. Cuenca terciaria del Alto Tajo. Hoja n560 (Alcalá de Henares). Instituto Geológico y Minero de España, Madrid.

Sancho-Gaspar, J.L. 1996. Análisis histórico del patrimonio pétreo. In: Mingarro, F. (ed.), Degradación y Conservación del Patrimonio Arquitectónico. Editorial Complutense, 311-316.

San José Lancha, M.A. 1975. Memoria Hoja $\mathrm{n}^{\circ} 583$ (Arganda). Mapa Geológico de España. Escala 1:50.000. Instituto Geológico y Minero de España, Madrid.

Sanz-Montero, M.E., Alonso-Zarza, A.M. and Calvo, J.P. 1995. Carbonate pond deposits related to semi-arid alluvial systems: examples from the Tertiary Madrid Basin, Spain: Sedimentology, 42, 437-452.

Sanz-Montero, M.E., Calvo, J.P, García del Cura, M.A. and Cañaveras, J.C. 2003. Rasgos morfológicos y petrológicos del paleokarst de la Unidad Superior del Mioceno de la Cuenca de Madrid. Estudios Geológicos, 59, 67-81.

Scholle, P.A. and Ulmer-Scholle, D.S. 2003. A Color Guide to the Petrography of Carbonate Rocks: Grains, textures, porosity, diagenesis. American Association of Petroleum Geologists Memoir, 77, 474 pp.

Schwenzner, J. 1936. Zur mophologie des Zentralspanischen Hochlandes. Geographische Abhandlungen. J. Engelhorns Nachfolger, Stuttgart, 129 pp.

Utanda, L. 2004. La evolución histórica en la comarca de las Vegas (1750-1950). In: Universitat de València (ed.), Historia, clima y paisaje: Estudios Geográficos en memoria del profesor Antonio López Gómez, 233-252.

UNE-EN 14579:2005. Métodos de ensayo para piedra natural. Determinación de la velocidad de propagación del sonido.

UNE-EN 1936:2007. Métodos de ensayo para piedra natural. Determinación de la densidad real y aparente y de la porosidad abierta y total.

UNE-EN 12407:2007. Métodos de ensayo para piedra natural. Estudio petrográfico.

UNE-EN 15802:2010. Conservación del patrimonio cultural. Métodos de ensayo. Determinación del ángulo de contacto estático.

Varas, M.J., Gomez-Heras, M. and Fort, R. 2003. Abastecimiento de piedra en monumentos de Madrid del siglo XIX: la Cripta de la Catedral de Santa María de la Almudena y el Panteón de Hombres llustres. Restauración y Rehabilitación, 79, 46-51. 
Varas-Muriel, M.J., Vázque-Calvo, C., Fort R. and PérezMonserrat, E.M. 2010. Canteras y monumentos históricos: un recurso didáctico. Enseñanza de Ciencias de la Tierra, 18 (3), 259-268.

Yasar, E. and Erdogan, Y. 2004. Estimation of rock physico- mechanical properties using hardness methods. Engineering Geology, 71, 281-288.

Zezza, U. 1990. Physical-mechanical properties of quarry and building stones. In: Veniale, F. and Zezza, U. (eds.), Advanced Workshop: Analytical Methodologies for the Investigation of Damaged Stones, Pavia, $21 \mathrm{pp}$.

Recibido: junio 2017

Revisado: junio 2017

Aceptado: julio 2017

Publicado: diciembre 2017 